\title{
Loop Representations for $2+1$ Gravity on a Torus
}

\author{
Donald M. Marolf \\ Physics Department, Syracuse University, Syracuse, New York 13244
}

(July 15, 2018)

\begin{abstract}
We study the loop representation of the quantum theory for $2+1$ dimensional general relativity on a manifold, $M=\mathcal{T}^{2} \times \mathcal{R}$, where $\mathcal{T}^{2}$ is the torus, and compare it with the connection representation for this system. In particular, we look at the loop transform in the part of the phase space where the holonomies are boosts and study its kernel. This kernel is dense in the connection representation and the transform is not continuous with respect to the natural topologies, even in its domain of definition. Nonetheless, loop representations isomorphic to the connection representation corresponding to this part of the phase space can still be constructed if due care is taken. We present this construction but note that certain ambiguities remain; in particular, functions of loops cannot be uniquely associated with functions of connections.
\end{abstract}

SU-GP-93/3-1,gr-qc/9303019

\section{INTRODUCTION}

In recent years the idea of a non-perturbative approach to quantum gravity has become increasingly popular. This is largely due to the fact that no satisfactory perturbative theory of quantum gravity has been found. Indeed, perturbative quantum gravity fails even by the usual standards of quantum field theory: that is, no renormalization scheme has been found that consistently makes the individual terms in the perturbation series finite. Efforts to extend quantum gravity using ideas such as introducing higher derivative terms in the action, requiring supersymmetry, etc., have failed as well.

Looking beyond the problems faced by these theories, a completely satisfactory perturbative formulation of a fundamental theory would have to do more than give a perturbation series whose individual terms are finite; it should give a series that actually converges. Even perturbative string theory does not meet this requirement [1]. As a result, the possibility that certain theories for which no perturbative quantization exists can be quantized using non-perturbative methods becomes attractive and the possibility that gravity itself is such a theory becomes even more so.

For these reasons interest in non-perturbative methods has grown and a number of non-perturbative approaches to quantizing gravity has been discussed. These include approaches that aim toward integrating numerically path integrals associated with general relativity both in a covariant field formulation [2] or in a Regge calculus style formulation [3]. Alternatively, nonperturbative canonical quantization has been considered using an ADM-based formalism to construct constraint operators that act on functions of three-metrics to select physical states [4].

A great simplification in the canonical approach is achieved by using a different set of variables to define the theory. Instead of considering metrics to be fundamental, this formulation is based on tetrads and self-dual connections [5.6]. This formulation defines constraint operators that are polynomial in the basic phase space coordinates so that these constraints may be much easier to impose to select physical states than the metric based constraints. In this formulation, it is the connection and not the tetrad that is considered to define the configurations space. One consequence of this change of viewpoint is that gauge invariant quantities in this formulation naturally involve holonomies around loops and their traces - the Wilson loop functionals - just as in other gauge theories.

In this way, loops enter naturally into this formulation of quantum gravity. It is even possible to define quantum states in terms of functions of loops. This set of loop functions provides a representation of operators also based on loops and is therefore called a "loop representation" [7, 9. Much effort has gone into the study of loop representations ranging from the explicit construction of an infinite dimensional space of solutions to the constraints [7] 11 , to the construction and regularization of the type of observables that are more familiar in general relativity and of states that reproduce certain properties of flat three dimensional spaces [8],9].

However, the program of quantizing gravity using a loop representation is far from complete and it is important to gain intuitive insight into the process as a whole by carrying out this program on simplified models and other simple systems. This allows us not only to gain perspective from which we can evaluate the program as a whole but also to take note of subtleties that may become important in trying to carry out this program in the full theory. In particular, loop representations have been constructed and analyzed for linear systems such as Maxwell fields [17 (U(1) connections) and linearized gravity $\sqrt{12}$ as well as for the genuinely nonlinear case of $2+1$ dimensional gravity on $\mathcal{T}^{2} \times \mathcal{R}$ 13, 14, 16]. We note, however, that the part of $2+1$ gravity on a torus that was analyzed in $\left.13,14,16\right]$ in terms 
of a loop representation describes only connections whose holonomies are rotations and not those whose holonomies are boosts. As a result, all of these models are based in effect on a compact gauge group in contrast to the full theory of $3+1$ gravity.

One of the objects studied in these simple models is the so-called "loop transform." This transform is a formal map from functions of connections to functions of loops. The investigation of the loop transform in these simple models is important because the loop transform has been frequently used, at least heuristically, in the study of the loop representation in the $3+1$ theory 179 . The idea is that it would send a state described by a function of connections to the same state's description in terms of functions of loops. One might hope that this loop transform could be used to show that suitable such loop representations are isomorphic to suitable connection representations and therefore to further justify the use of loop representations. These expectations are borne out in simple models including $2+1$ gravity on a torus in the "sector" in which the holonomies are rotations. There has also been a general attempt to make the loop transform and well defined using Gel'fand spectral theory [18].

Our purpose here will be to reopen the study of the loop representations of $2+1$ gravity on a torus by concentrating instead on the part of the theory where the holonomies are boosts. This is important from a physical point of view, since it is this part of the phase space that actually corresponds to spacetimes in which the toroidal sections are spacelike surfaces [23, 15]. It is also important from the point of loop representations since this part of the theory is a simple model with a non-compact gauge group. We will see that this leads to a quite different loop representation as well as a distinct change in the properties of the loop transform.

This paper begins with a description of classical $2+1$ general relativity formulated in terms of a connection and a triad. In the spirit of canonical quantization, we examinethe canonical structure of this theory and a description of the reduced phase space. In particular, we look at the "sector" of the phase space in which the holonomies are all rotations and the one where the holonomies are all boosts. With this description in terms of holonomies, we then introduce the loop-dependent phase space functions that define the classical "loop algebra." This is the algebra that leads to loop representations in the quantum theory.

Using this classical description, we can define a quantum theory using reduced phase space quantization in both a connection representation and a loop representation for both of the above sectors of the phase space. As mentioned before, the loop transform is a representation isomorphism between connection and loop representations in the sector in which the holonomies are rotations. However, as we demonstrate in Appendix D, the naively defined loop representation in the sector where the holonomies are rotations is identical to the one defined in the sector where the holonomies are boosts. This result is disturbing since the connection representations and even the classical solutions corresponding to these sectors have quite different properties.

To understand this issue, we investigate the loop transform in the sector where the holonomies are boosts. As might be expected from our dilemma, the loop transform is no longer a representation isomorphism in this sector. Instead, the loop transform has a non-trivial kernel which turns out to be so large that it is dense in the connection representation. The loop transform is also far from continuous as a map from the connection representation to functions of loops. The proof of these statements represents a digression from the main discussion and is therefore relegated to Appendix A.

Despite these problems, we then show that loop representations isomorphic to the connection representation can in fact be constructed in this sector, though this requires extra care. The construction of such loop representations is based on the choice of a dense subspace of the connection representation that satisfies a short list of properties. The actual construction of such subspaces again represents a tangent to the main discussion and is presented in Appendix B. Since each of these representations is isomorphic to the connection representation, this construction is independent of the subspace chosen up to isomorphism. Nonetheless, certain ambiguities remain in the loop representation and we discuss these as well. We note that many of these ambiguities can be removed by extending our representation from functions of loops to functions of so-called "generalized loops." Finally, we close with a analogy between the loop transform and the so-called "Mellin Transform" and a short discussion of the possibility of extrapolating our results to $2+1$ gravity on on other compact spacial slices and to the full theory in $3+1$ dimensions.

\section{SUMMARY OF THE CLASSICAL FORMULATION}

We now begin with a brief review of the classical connection formulation of $2+1$ dimensional general relativity following [16]. This will include a review of the canonical description which we will use in section [II for canonical quantization of this system. Our canonical discussion will illustrate the construction of the reduced phase space and its division into "sectors" on which we will base our quantum theory. This quantum theory will be described in two ways: in terms of a connection representation and in terms of a "loop representation." This loop representation is defined as a representation of the so-called "loop algebra" and therefore we we will also introduce the classical 
functions that define this algebra. We will see that these functions can be used to label points in the two phase space sectors on which we will concentrate.

Our classical description begins with the basic variables of our theory; the co-triads, ${ }^{3} e_{a}^{I}$, and $S U(1,1)$ connections, ${ }^{3} A_{a}^{I}$. Instead of $S U(1,1)$, we could use $S O(2,1)$ or its connected component as our gauge group without significantly changing the discussion in sections III- VI. [15] These variables are to be subject to the dynamics specified by the action:

$$
S\left({ }^{3} e_{a}^{I},{ }^{3} A_{b}^{I}\right)=\int_{M} d^{3} x \tilde{\eta}^{a b c}{ }^{3} e_{a}^{I}{ }^{3} F_{b c I}
$$

where $\tilde{\eta}^{a b c}$ is the Levi-Civita density on a three-dimensional manifold $M$ and

$$
{ }^{3} F_{a b I}=2 \partial_{[a}{ }^{3} A_{b] I}+\epsilon_{I J K}{ }^{3} A_{a}^{J 3} A_{b}^{K}
$$

is the curvature of the connection, ${ }^{3} A_{b}^{I}$. Internal indices, $\mathrm{I}, \mathrm{J}, \ldots$, are raised and lowered using the $2+1$ Minkowski metric. This action is analogous to the so-called "self-dual action" in $3+1$ gravity [21]. As in that case, one can allow the triad to become degenerate, although for nondegenerate triads the theory is equivalent to $2+1$ general relativity.

The dynamics are described more transparently through the classical equations of motion:

$$
{ }^{3} \mathcal{D}_{[a}{ }^{3} e_{b] I}=0 \text { and }{ }^{3} F_{a b}^{I}=0
$$

Here, ${ }^{3} \mathcal{D}$ is the gauge covariant derivative operator determined by ${ }^{3} A_{a}^{I}$, so that these equations just say that the connection is compatible with the triad and than the curvature of this connection is flat. Note that, as defined, ${ }^{3} \mathcal{D}$ acts only on internal indices. We could extend $\mathcal{D}$ to act on spacetime indices in a number of ways, such as by requiring compatibility with the triad, though this is not necessary.

Since we are interested in a canonical formulation of this theory, we now consider the Legendre transform of the above action. We assume that $M$ is of the form $\Sigma \times \mathcal{R}$ and take the space of pull-backs, $A_{a}^{I}$, of the connection, ${ }^{3} A_{a}^{I}$, to a spacial slice, $\Sigma$, to be our the configuration space. The momenta, $\tilde{E}_{I}^{a}$, are then duals of the pull-backs of the co-triads: $\tilde{E}_{I}^{a}=\tilde{\eta}^{a b} e_{b I}$, where $\tilde{\eta}^{a b}$ is the anti-symmetric Levi-Civita density on $\Sigma$ and $e_{b I}$ is the pull-back of ${ }^{3} e_{b I}$ to $\Sigma$. The other variables are Lagrange multipliers that enforce the constraints:

$$
\mathcal{D}_{a} \tilde{E}_{I}^{a}=0 \text { and } F_{a b}^{I}=0
$$

where $F_{a b}^{I}$ is the curvature of $A_{a}^{I}$ and $\mathcal{D}$ is this connection's covariant derivative operator.

The first constraint in 2.4 generates internal $S U(1,1)$ transformations while the second generates diffeomorphisms and the usual Hamiltonian gauge transformation in $2+1$ gravity. This second constraint actually generates an extension of these transformations to degenerate triads. Note that this second constraint depends only on the connection and not on the conjugate momenta. It follows that any function of connections is invariant under transformations generated by this constraint and that any function of connections that is invariant under internal gauge transformations is a Dirac observable and has vanishing Poisson Brackets with all constraints.

We will use such gauge invariant functions of connections to label the reduced configuration space of our system. This will allow us to describe the reduced phase space since the reduced phase space will be the cotangent bundle over the reduced configuration space. From Eq. 2.4, we see that our reduced configuration space is the space of $S U(1,1)$ equivalence classes of flat connections on $\Sigma$.

A gauge equivalence class of connections on $\Sigma$ can be characterized by its (equivalence class of) holonomies, $U(\alpha)$, around all closed loops $\alpha$. Holonomies have much less gauge freedom than connections and so provide a step toward a reduced configuration space description. Since the connection is flat, the holonomies around all homotopically trivial loops are the identity transformation and $U(\alpha)$ depends only on the homotopy class, $[\alpha]$, of $\alpha$. This set of holonomies forms a homomorphism from the homotopy group to $S U(1,1)$ since $U([\beta] \circ[\gamma])=U([\beta]) U([\gamma])$.

The homotopy group of our surface, the torus, is abelian with $\pi_{1}\left(\mathcal{T}^{2}\right)=\mathcal{Z} \oplus \mathcal{Z}$. Since the set of holonomies forms a homomorphism, it follows that for all closed loops $\alpha, U([\alpha])$ is determined by the holonomies around the two generators, $\alpha_{1}$ and $\alpha_{2}$, of $\pi_{1}\left(\mathcal{T}^{2}\right)$ and that $U\left(\left[\alpha_{1}\right]\right)$ and $U\left(\left[\alpha_{2}\right]\right)$ commute. Therefore, gauge equivalence classes of flat connections on the torus are labeled by pairs of commuting $S U(1,1)$ transformations.

We would like to find a simple parameterization of these holonomies. Note that each holonomy $U\left[\alpha_{b}\right]$ is an $S U(1,1)$ transformation that leaves invariant some internal $2+1$ Lorentz vector $A_{b}^{I}$ and that an infinitesimal transformation that leaves this vector invariant is generated by the element $A_{b}^{I} \tau_{I}$ in the Lie algebra of $S U(1,1)$. Here, $\left\{\tau_{I}\right\}$ is basis for this Lie algebra that satisfies:

$$
\left[\tau_{0}, \tau_{1}\right]=-i \frac{1}{2} \tau_{2}
$$




$$
\begin{gathered}
{\left[\tau_{2}, \tau_{0}\right]=-i \frac{1}{2} \tau_{1}} \\
{\left[\tau_{1}, \tau_{2}\right]=i \frac{1}{2} \tau_{0}}
\end{gathered}
$$

Some finite transformations that leave this vector invariant are given by

$$
g=\exp \left(\lambda A_{b}^{I} \tau_{I}\right)
$$

for $\lambda \in \mathcal{R}$.

Unfortunately, not all elements of $S U(1,1)$ can be written as the exponential of some element of the Lie Algebra [15]. If a transformation preserves a timelike vector, then it can be written in the form of Eq. 2.6 for appropriate $\overrightarrow{A_{b}^{I}}$ but this is not necessarily true if the transformation preserves a spacelike or null vector. If the transformation preserves a spacelike or null vector, then this transformation can either be written in the form of Eq. 2.6 or it can be written as an element of the form given in Eq. 2.6 multiplied by the group element $R(2 \pi) . R(2 \pi)$ is the group element that when acting on spinors induces a rotation through the angle $2 \pi$ about any spacelike axis. In the two-dimensional representation of $S U(1,1) R(2 \pi)$ is represented by the matrix $-\mathbb{1}$. Because of this, and because $R(2 \pi)$ commutes with all $g \in S U(1,1)$, we will label group elements which when acting on vectors preserve some null or spacelike vector by the pair

$$
g_{b}=\left(A_{b}^{I}, \epsilon_{b}\right)=\exp \left(A_{b}^{I} \tau_{I}\right)[R(2 \pi)]^{\left(\frac{\epsilon_{b}-1}{2}\right)}
$$

All elements of $S U(1,1)$ for which $A_{b}^{I}$ is spacelike or null are uniquely labeled by this prescription. Group elements that preserve a timelike vector can also be labelled in this way but do not require the extra index, $\epsilon_{b}$.

We would now like to see what restrictions we place on our parameters when we require that the holonomies commute. Two $S U(1,1)$ transformations commute only if they both preserve the same $2+1$ dimensional vector so $A_{1}^{I}$ and $A_{2}^{I}$ must both be proportional to the same internal vector, $t^{I}$ :

$$
A_{b}^{I}=2 a_{b} t^{I}
$$

The $\epsilon_{b}$ labels that arise when $t^{I}$ is spacelike or null are unrestricted by this requirement.

The next step is to translate the gauge transformations of the holonomies into transformations on these internal vectors. The sets of holonomies fall into equivalence classes under gauge transformations which act on the set of holonomies by conjugation with an element of $S U(1,1)$ :

$$
\{U[\alpha]\} \rightarrow\left\{g U[\alpha] g^{-1}\right\}
$$

Since $R(2 \pi)$ commutes with every $S U(1,1)$ transformation, this will have no effect on the labels $\epsilon_{b}$ that may be required. On the other hand, conjugation of $\exp \left(A_{b}^{I} \tau_{I}\right)$ by the group element, $g$, yields the element, $\exp \left(\tilde{A}_{b}^{I} \tau_{I}\right)$, where $\tilde{A}_{b}^{I}$ is obtained from $A_{b}^{I}$ by the action of the group element $g$ on internal vectors since our internal vectors label elements of the Lie algebra of $S U(1,1)$. Thus, equivalence classes of pairs of holonomies are labeled by equivalence classes of pairs of parallel internal vectors under $S U(1,1)$ transformations.

To classify these equivalence classes completely note that for timelike or spacelike $t$ we can choose $a_{b}$ so that the norm of $t$ is \pm 1 . Also note that for $t$ null or timelike we can always choose $a_{b}$ to have appropriate signs so that $t$ is future directed. Now, all normalized future directed timelike $t$ 's are related by $S U(1,1)$ transformations, as are all normalized spacelike $t$ 's and all future directed null $t$ 's. Following Ref. [16] we will refer to the regions of reduced configuration space corresponding to these three equivalence classes as the timelike, spacelike, and null "sectors" of the theory and proceed to consider them separately. (For further details, see Ref. 115]).

In each of these sectors, we would like to find an explicit parameterization of the configuration space. Suppose that, in each sector, we fix a representative internal vector $t^{I}$ subject to the above conditions. Note that in the spacelike sector the holonomies are boosts so that each $a_{b}$ in $\mathcal{R}$ labels a distinct $S U(1,1)$ transformation, $\left(2 a_{b} t^{I}, \epsilon_{b}\right)$. However, given any spacelike vector, $t^{I}$, there is an $S U(1,1)$ transformation that can reverse its direction. Thus we can change $\left(2 a_{b} t^{I}, \epsilon_{b}\right)$ into $\left(-2 a_{b} t^{I}, \epsilon_{b}\right)$ by a gauge transformation and, since we defined our parameterization by regarding $t^{I}$ as fixed, we should identify $\left\{a_{b}\right\}$ and $\left\{-a_{b}\right\}$ as gauge related. No other values of these parameters give rise to gauge related holonomies so the reduced configuration space in the spacelike sector is labeled by $\left\{a_{b}\right\} \in \mathcal{R}^{2} / \mathcal{Z}^{2}$ and $\left\{\epsilon_{b}\right\} \in\{-1,1\}$.

To find the reduced configuration space in the null sector, we note that the $S U(1,1)$ transformations $\exp \left(2 a_{b} t^{I} \tau_{I}\right)$ are distinct for $\left\{a_{b}\right\} \in \mathcal{R}^{2}$. However, we must identify them in gauge equivalence classes under $S U(1,1)$ transformations. $S U(1,1)$ transformations can scale any null vector $2 a_{b} t^{I}$ by any positive real number but leave the labels $\epsilon_{b}$ unaffected. 
If we ignore the equivalence class labeled by $\left\{a_{b}\right\}=0$ then since $t^{I}$ is fixed each equivalence class is uniquely labeled by an angular coordinate. The reduced configuration space for the null sector is thus $S^{1} \times\left(\mathcal{Z}_{2}\right)^{2}$ together with four additional points representing the equivalence classes corresponding to the pairs of holonomies $( \pm \mathbb{1}, \pm \mathbb{1})$.

Finally, in the timelike sector, the holonomies are rotations about some axis. Distinct rotations in $S U(1,1)$ are labeled by angles defined modulo $4 \pi$. For a rotation, $\exp \left(\theta \tau_{0}\right)$ around the 0 -axis, this angle is just the coefficient, $\theta$. If we fix $t^{I}$ in the timelike sector to point along the 0 -axis, it follows that our labels $a_{1}$ and $a_{2}$ in the configurations space are both periodic with period $2 \pi$ and that the configuration space is $a_{b} \in \mathcal{T}^{2}$. Note that all of these sectors overlap at the zero connection $\left(a_{b}=0\right)$. The zero connection represents a special point in phase space, which will be discussed in [15]. This represents a technical point that we ignore here because it does not substantially alter the discussion.

We are interested only in the spacelike and timelike sectors and, following [16], we take the corresponding reduced phase spaces to be the cotangent bundles over $\mathcal{R}^{2}$ and $\mathcal{T}^{2}$ respectively. This means that we include the zero connection in both sectors and deal with the $\mathcal{Z}_{2}$ symmetry of the spacelike sector separately in order to give the reduced phase space the structure of a cotangent bundle over a manifold without boundary $\$$. This ensures that the resulting quantum treatment of the spacelike sector is the one that would result from Dirac quantization. Because we are treating the sectors as disconnected and the four spacelike sectors (labeled by the $\epsilon_{b}$ ) are similar, we will consider only the spacelike sector for which the discrete indices take the values $\epsilon_{1}=+1=\epsilon_{2}$. This completes the characterization of the reduced configuration space that we have been working toward.

One final element of the classical theory that we will need for our quantum description is the set of functions that make up the classical "loop algebra" [8,16]. The loop algebra is defined by the Poisson algebra of the functions:

$$
\begin{gathered}
\mathcal{T}^{0}[\alpha](A)=\frac{1}{2} \operatorname{Tr} U(\alpha) \\
\mathcal{T}^{1}[\alpha](A, \tilde{E})=\oint_{\alpha} d S^{a} \operatorname{Tr} E_{a} U(\alpha)
\end{gathered}
$$

for all closed loops, $\alpha$, where $E_{a}=\bar{\eta}_{a b} \tilde{E}^{b}$ ( $\bar{\eta}$ is the anti-symmetric density on $\Sigma$ of weight -1$)$ and the 2-dimensional representation of $S U(1,1)$ is used to take the trace. It can be shown on general grounds that these functions are overcomplete almost everywhere on the phase space of connections identified in gauge equivalence classes under internal gauge transformations and so can be used to label points in this phase space up to a small ambiguity [28].

Although these functions take arbitrary closed loops as one of their arguments, we are considering only flat connections and these functions therefore depend only on homotopy classes of loops. Since any homotopy class $[\alpha]$ can be decomposed in terms of the generators, $[\alpha]=n_{1}\left[\alpha_{1}\right]+n_{2}\left[\alpha_{2}\right]$, it will be simplest to regard the $\mathcal{T}^{0}$ 's and $\mathcal{T}^{1}$ 's as functions of pairs of integers, $\mathbf{n}=\left(n_{1}, n_{2}\right)$. Eq. 2.10 shows that the $\mathcal{T}^{0}$ 's and $\mathcal{T}^{1}$ 's are all invariant under reversal of the loops so that distinct $\mathcal{T}^{0}$ 's and $\mathcal{T}^{1}$ 's are actually labelled by $\mathbf{n} \in \mathcal{Z}^{2} / \mathcal{Z}_{2}$.

Using this notation, we can write the Poisson algebra of the $\mathcal{T}^{0}$ 's and $\mathcal{T}^{1}$ 's as

$$
\begin{gathered}
\left\{\mathcal{T}^{0}(\mathbf{k}), \mathcal{T}^{0}(\mathbf{m})\right\}=0 \\
\left\{\mathcal{T}^{0}(\mathbf{k}), \mathcal{T}^{1}(\mathbf{m})\right\}=i \mathbf{k} \times \mathbf{m}\left(\mathcal{T}^{0}(\mathbf{k}+\mathbf{m})-\mathcal{T}^{0}(\mathbf{k}-\mathbf{m})\right) \\
\left\{\mathcal{T}^{1}(\mathbf{k}), \mathcal{T}^{1}(\mathbf{m})\right\}=i \mathbf{k} \times \mathbf{m}\left(\mathcal{T}^{1}(\mathbf{k}+\mathbf{m})-\mathcal{T}^{1}(\mathbf{k}-\mathbf{m})\right)
\end{gathered}
$$

where $\mathbf{k} \times \mathbf{p}=k_{1} p^{2}-k_{2} p^{1}$. The $\mathcal{T}^{0}$ 's and $\mathcal{T}^{1}$ 's also obey the relations

$$
\begin{gathered}
\mathcal{T}^{0}(\mathbf{k}) \mathcal{T}^{0}(\mathbf{m})=\frac{1}{2}\left(\mathcal{T}^{0}(\mathbf{k}+\mathbf{m})+\mathcal{T}^{0}(\mathbf{k}-\mathbf{m})\right) \\
\mathcal{T}^{0}(\mathbf{k}) \mathcal{T}^{1}(\mathbf{m})+\mathcal{T}^{0}(\mathbf{m}) \mathcal{T}^{1}(\mathbf{k})=\frac{1}{2}\left(\mathcal{T}^{1}(\mathbf{k}+\mathbf{m})+\mathcal{T}^{1}(\mathbf{k}-\mathbf{m})\right)
\end{gathered}
$$

Note that the $\mathcal{T}^{0}$ 's are functions of connections only and that that are invariant under internal gauge transformations. They are therefore invariant under all gauge transformations and describe functions on the reduced configuration space. The $\mathcal{T}^{1}$ 's are invariant under internal gauge transformations but involve the momenta as well as the connection

\footnotetext{
${ }^{1}$ For a proper treatment of the reduced phase space, see [15].
} 
so that their Poisson Brackets with the the $F_{a b}^{I}=0$ constraint must be checked. However, explicit calculation shows that these Poisson Brackets vanish. It follows that the $\mathcal{T}^{1}$ 's are also functions on the reduced phase space. Both $\mathcal{T}^{0}$ 's and $\mathcal{T}^{1}$ 's can therefore be written in terms of the phase space coordinates described above:

$$
\begin{gathered}
\mathcal{T}^{0}(\mathbf{k})=\cos (\mathbf{k} \cdot \mathbf{a}) \\
\mathcal{T}^{1}(\mathbf{k})=(\sin (\mathbf{k} \cdot \mathbf{a})) \mathbf{k} \times \mathbf{p}
\end{gathered}
$$

in the timelike sector and by

$$
\begin{gathered}
\mathcal{T}^{0}(\mathbf{k})=\cosh (\mathbf{k} \cdot \mathbf{a}) \\
\mathcal{T}^{1}(\mathbf{k})=(\sinh (\mathbf{k} \cdot \mathbf{a})) \mathbf{k} \times \mathbf{p}
\end{gathered}
$$

in the spacelike sector, where the bold-faced letters represent two component vectors and $p^{b}$ is the momentum conjugate to $a_{b}$.

Note that because the $\mathcal{T}^{0}$ 's and $\mathcal{T}^{1}$ 's are invariant under $\mathbf{a} \rightarrow-\mathbf{a}$, they are two-fold degenerate in both sectors. This will reintroduce the desired $\mathcal{Z}_{2}$ symmetry in the spacelike sector and lead to a split in the quantum theory of the connection representation in the timelike sector into two irreducible representations of the $\mathcal{T}^{0}, \mathcal{T}^{1}$ algebra. Other than this, we see that the $\mathcal{T}^{0}$ 's and $\mathcal{T}^{1}$ 's are indeed an overcomplete set of functions on the reduced phase space except at the zero connection in the spacelike sector and at $a_{b} \in\{0, \pi\}$ in the timelike sector.

This completes our description of the classical reduced phase space. We see that it can be considered to have several "sectors," two of which are the "timelike" sector, in which the fundamental holonomies are two commuting rotations and the "spacelike" sector in which the fundamental holonomies are two commuting boosts. The reduced configuration space is a torus in the timelike sector and a plane in the spacelike sector. In each sector, we introduced additional functions on the phase space, $\mathcal{T}^{0}, \mathcal{T}^{1}$, for each homotopy class that define the classical loop algebra and can be used to label points on the reduced phase space up to a small number of degeneracies.

\section{THE QUANTUM THEORY}

We could build a quantum theory using either reduced phase space quantization or Dirac constraint quantization. In this case, (with proper choices of factor ordering) we would find equivalent results. (Compare our results with [16].) Because the above constraints are linear in or independent of momenta, this is what would be expected (see, eg. [19]), up to questions about the gauge orbits. We will choose reduced phase space quantization, which will allow us to use the classical discussion of the last section to construct a connection representation of the quantum theory in a straightforward manner.

A connection representation is defined as a representation of some algebra on a Hilbert space of functions of connections. Since our equivalence classes of connections are labeled in each sector by $\left\{a_{b}\right\}$ we will define our connection representation to consist of functions of $a_{b}$ that are square integrable with respect to some measure. This measure must be determined by some additional requirements. If we wish to construct a representation of $a_{b}$ and $p^{b}$ then it is reasonable to to require $a_{b}$ and $p^{b}$ to be Hermitian from which it follows that the measure must be $d \mu=d^{2} a$. If we take the $\mathcal{T}^{0}$ 's and $\mathcal{T}^{1}$ 's to be given by

$$
\begin{gathered}
\mathcal{T}^{0}(\mathbf{k})=\cos (\mathbf{k} \cdot \mathbf{a}) \\
\mathcal{T}^{1}(\mathbf{k})=(\sin (\mathbf{k} \cdot \mathbf{a})) \mathbf{k} \times\left(-i \frac{\partial}{\partial \mathbf{a}}\right)
\end{gathered}
$$

in the timelike sector and by

$$
\begin{gathered}
\mathcal{T}^{0}(\mathbf{k})=\cosh (\mathbf{k} \cdot \mathbf{a}) \\
\mathcal{T}^{1}(\mathbf{k})=(\sinh (\mathbf{k} \cdot \mathbf{a})) \mathbf{k} \times\left(-i \frac{\partial}{\partial \mathbf{a}}\right)
\end{gathered}
$$


in the spacelike sector, then the $\mathcal{T}^{0}, \mathcal{T}^{1}$ 's are also Hermitian with respect to this inner product ${ }^{2}$. With this ordering, the $\mathcal{T}^{0}$ 's and $\mathcal{T}^{1}$ 's again satisfy Eq. 2.12 and their commutation relations are given by $i$ times the Poisson brackets ordered as in Eq. 2.11. Thus, the Hilbert space of these connection representations also carries a representation of the quantum loop algebra with Hermitian $\mathcal{T}^{0}$ 's and $\mathcal{T}^{1}$ 's. In fact, this same Hilbert space would arise if we were to define the space as a connection representation of the loop algebra (see Appendix Q).

Note that the $\mathcal{T}^{0}, \mathcal{T}^{1}$ algebra preserves the various sectors. Each sector must therefore provide a representation of this algebra. Because the $\mathcal{T}^{0}$ 's and $\mathcal{T}^{1}$ 's can be used to construct the action of $\mathbf{a}$ and $\mathbf{p}$ on a dense set of states in the spacelike sector of the connection representation, no closed linear subspace of the carrier space of the connection representation in the spacelike sector is left invariant by the action of the $\mathcal{T}^{0}$ 's and $\mathcal{T}^{1}$ 's. In other words, the spacelike connection representation forms a topologically irreducible representation of the loop algebra. However, in the timelike sector this construction is possible only up to an ambiguity between $\mathbf{a}$ and $-\mathbf{a}$. As a result, the timelike sector contains two topologically irreducible representations of the loop algebra. One of these is carried by the set of functions that are symmetric under $\mathbf{a} \rightarrow-\mathbf{a}$ and the other is carried by the set of functions that are antisymmetric under this operation. We will henceforth refer to these sets of functions as the symmetric and antisymmetric timelike sectors.

This completes our discussion of the connection representations. Indeed, our goal is to examine the so-called loop representation so we now proceed in this direction. We begin with the definition of a loop representation: a loop representation is a representation of the $\mathcal{T}^{0}, \mathcal{T}^{1}$ algebra on a Hilbert space which is obtained by completion in the topology defined by some inner product of some linear space whose elements are suitable functions of loops in a manifold $2^{3}$. Usually, this linear space is taken to be the set of functions with support on a finite number of loops.

"Suitable" functions of loops are to be constant on equivalence classes of loops defined as follows: two closed loops $\alpha$ and $\beta$ are equivalent if and only if $\operatorname{Tr} U[\alpha](A)=\operatorname{Tr} U[\beta](A)$ for all connections $A$ in whatever class of connections we are considering 16. These equivalence classes are called equitopy classes. Since we are concerned here only with flat connections on $\overline{\mathcal{T}}^{2}$ and since for this class of connections $U(\alpha)$ depends only on the homotopy class of $\alpha$, our representation will be in terms of functions of homotopy classes of loops in $\mathcal{T}^{2}$. In addition, inverting a loop $(\alpha \rightarrow-\alpha)$ does not change the trace of the holonomy around it. Thus, we consider functions of loops that are invariant under inversion of loops. Such functions are completely characterized by a function $\psi: \mathcal{R}^{2} / \mathcal{Z}_{2} \rightarrow \mathcal{C}$.

These functions of loops carry a representation of the $\mathcal{T}^{0}(\mathbf{k}), \mathcal{T}^{1}(\mathbf{k})$ 's, which are taken to be the fundamental operators in this "loop representation". The action of these operators on functions of loops is given by:

$$
\begin{gathered}
\left(\mathcal{T}^{0}(\mathbf{k}) \psi\right)(\mathbf{n})=(\psi(\mathbf{n}+\mathbf{k})+\psi(\mathbf{n}-\mathbf{k})) / 2 \\
\left(\mathcal{T}^{1}(\mathbf{k}) \psi\right)(\mathbf{n})=i \mathbf{k} \times \mathbf{n}(\psi(\mathbf{n}+\mathbf{k})-\psi(\mathbf{n}-\mathbf{k}))
\end{gathered}
$$

With these definitions, the $\mathcal{T}^{0}$ 's and $\mathcal{T}^{1}$ again satisfy commutation relations given by $i$ times the Poisson brackets in Eq. 2.11 and the algebraic relations given in Eq. 2.12.

The loop representation can be formally related to the connection representation via the "loop transform" (see Ref. [7]) which defines a function of loops from a function of connections. The loop transform has the general form:

$$
\psi(\alpha) \equiv \int d \mu(A) \psi(A) \mathcal{T}^{0}[\alpha](A)
$$

which, in our case, is just

$$
\psi(\mathbf{n})=\int d^{2} a \psi(\mathbf{a}) \mathcal{T}^{0}(\mathbf{n})
$$

Formal arguments indicate in general that the loop transform maps $\mathcal{T}^{0}$ 's and $\mathcal{T}^{1}$ 's in the connection representation to $\mathcal{T}^{0}$ 's and $\mathcal{T}^{1}$ 's in the loop representation [7]. These arguments are rigorous in the timelike and spacelike sectors when these operators act on functions $\psi(\mathbf{a})$ of connections on which the loop transform is defined or functions $\hat{\psi}(\mathbf{n})$ of loops in the image of the loop transform.

\footnotetext{
${ }^{2}$ In equations 3.1 and 3.2 we have chosen units such that $\hbar=1$ and we will continue to chose such units throughout our discussion.

${ }^{3}$ This is a consequence of the definition given in 16 .

${ }^{4}$ In the rest of this section and the next, two equitopy classes of loops will be referred to simply as "loops."
} 
While the loop transform can be used to relate the connection and loop representations, this relationship takes different forms in different sectors. For example, the $\mathcal{T}^{0}(\mathbf{k})$ 's are symmetric under inversion of loops so that the loop transform annihilates any function of connections in the antisymmetric timelike sector. However, in the symmetric timelike sector, the loop transform is just the Fourier transform so that if we impose the $l^{2}$ inner product on the functions of equitopy classes of loops, the loop transform is a representation isomorphism. In fact, the $l^{2}$ inner product is the only inner product on the functions of loops for which $\mathcal{T}^{0}, \mathcal{T}^{1}$ 's are Hermitian and the representation is topologically irreducible if we suitably restrict what we mean by a loop representation. In particular we show in Appendix D that this follows if we require the zero function of loops to represent the zero state and the representation to contain at least one function with support on a single loop. In this sense, the most natural inner product to impose on a loop representation gives a representation which is isomorphic to the connection representation in the symmetric timelike sector.

We would like to ensure that the representations of the $\mathcal{T}^{0}$ and $\mathcal{T}^{1}$ operators in a loop representation capture enough of the properties of the classical $\mathcal{T}^{0}$ and $\mathcal{T}^{1}$ functions that we would have some hope that a quantum theory based on this representation could produce the correct classical limit. In particular, classically the $\mathcal{T}^{0}$ and $\mathcal{T}^{1}$ functions are subject to a number of relations that include both linear relations and inequalities (see [29]). Quantum versions of these relations appear in the connection representation. Yet, it is difficult to see how we could ensure that anything like this set of relations appears in a loop representation, unless we know that the loop representation is isomorphic to a connection representation. For this purpose and others (see [8.7]) it would be nice if the loop transform was always a representation isomorphism, at least when the $\mathcal{T}^{0}$ 's and $\mathcal{T}^{1}$ 's form an almost complete set of operators as they do in the spacelike and symmetric timelike sectors. In such cases, we could then do away with the connection representation entirely and use only the loop representation. Let us suppose for a moment that this is true.

Notice that the loop representation is exactly the same in both the spacelike and timelike sectors. Both sectors lead to the representation (3.3) of the loop algebra as well as to the same definitions of equitopy classes of loops and therefore to the same class of functions of loops. As indicated above, the $l^{2}$ inner product is the only inner product in this representation compatible with the assertion that $\mathcal{T}^{0}$ 's and $\mathcal{T}^{1}$ 's are Hermitian, as they are in both the spacelike and timelike connection representations, and with the assertions that the representation is topologically irreducible, contains a function with support on a single loops, and is such that the zero function of loops represents the zero state. Unless there is additional input, there is no remaining freedom to construct a loop representation. If, then, the loop transform is a representation isomorphism in both sectors, all of the above requirements (except perhaps the existence of a function with support on a single loop) would be satisfied in both sectors and we would expect the two sectors to be isomorphic.

This result is disturbing. Not only do the two sectors have extremely different properties in terms of classical spacetimes (see [15]), but the quantum theories in the connection representation are distinct as well. To see this, note that in both representations, $\mathcal{T}^{0}(\mathbf{k})$ are multiplication operators. However, in the timelike sector they are represented by multiplication by functions of absolute value equal to or less than one whereas in the spacelike sector they are represented by multiplication by functions of absolute value equal to or greater than one. Thus the spectrum $\sigma\left(\mathcal{T}^{0}(\mathbf{k})\right)$ of $\mathcal{T}^{0}(\mathbf{k})$ is bounded in the timelike sector $\left(\sigma\left(\mathcal{T}^{0}(\mathbf{k})\right)=[-1,1]\right)$, but not in the spacelike sector $\left(\sigma\left(\mathcal{T}^{0}(\mathbf{k})\right)=[1, \infty)\right.$ for the $\epsilon_{1}=+1=\epsilon_{2}$ spacelike sector). These representations are certainly not isomorphic. We therefore expect trouble with the loop transform in the spacelike sector.

\section{THE LOOP TRANSFORM IN THE SPACELIKE SECTOR}

We saw in the preceding section that the loop transform has different properties in different sectors of the theory. For example, it annihilates the antisymmetric timelike sector but is a representation isomorphism in the symmetric timelike sector. We also took a brief look at a dilemma that could arise if the loop transform is a representation isomorphism in the spacelike sector. Therefore, we would now like to examine the loop transform more carefully in this sector. From this, we should learn about the loop transform in general and about the resolution of our dilemma in particular.

For a careful study, we will need the explicit form of the loop transform, $\mathbf{L}$, in the spacelike sector:

$$
(\mathbf{L}(\psi))(\mathbf{n})=\int d^{2} a \cosh (\mathbf{n} \cdot \mathbf{a}) \psi(\mathbf{a})
$$

\footnotetext{
${ }^{5}$ Thanks to Jorma Louko for pointing this out.
} 
Note that $\mathbf{L}$ is just the (two-sided) Laplace transform in this sector due to the symmetry of the functions of connections. Explicitly, $\mathbf{L}$ is the two-sided Laplace transform in each coordinate, $\left(a_{1}, a_{2}\right)$ :

$$
(\mathbf{L} \psi)(\mathbf{n})=\int_{-\infty}^{\infty} d a_{1} \int_{-\infty}^{\infty} d a_{2} e^{n_{1} a_{1}} e^{n_{2} a_{2}} \psi(\mathbf{a}),
$$

evaluated at integer points, $\mathbf{n} \in \mathcal{Z}^{2}$.

Unfortunately, $\mathbf{L}$ is not defined for all symmetric $\mathcal{L}^{2}(\mathcal{R})$ functions ${ }^{6}$. Let $\mathcal{D}_{\mathbf{L}}$ be the domain in $\mathcal{L}_{+}^{2}$ on which $\mathbf{L}$ is defined. Note that $\mathcal{D}_{\mathbf{L}}$ is a linear space that is dense in $\mathcal{L}_{+}^{2}$ as it contains all $C^{\infty}$ functions of compact support.

At this point, a theorem about inverting the two-sided Laplace transform will prove useful to our discussion. From Ref. [22], we find that if a holomorphic function $\psi_{*}(s)$ defined on the complex plane satisfies the following requirements:

i) For each $s \in \mathcal{R}$, there is some $M_{s} \in \mathcal{R}$ such that $\left|\int_{-\infty}^{\infty} d t \psi_{*}(s+i t)\right|<M_{s}$, and

ii) The limit, $\lim _{|t| \rightarrow \infty} \psi_{*}(s+i t)$ converges uniformly to zero for $t$ in any closed interval in the real line,

then there is some function, $\psi(a)$, such that $\psi_{*}$ is the two-sided Laplace transform of $\psi$. The two functions are related by

$$
\psi(a)=\int_{-\infty}^{\infty} d t \psi_{*}(i t) \exp (-i t a) \quad \text { and } \quad \psi_{*}(\mathrm{~s})=\int_{-\infty}^{\infty} \text { da } \psi(\mathrm{a}) \exp (\mathrm{as})
$$

Thus $\psi_{*}(i t)$ is just the Fourier transform of $\psi(a)$ if the above conditions are satisfied.

We will continue to use this notation: Bold arguments take values in a two dimensional space while normal print arguments take one dimensional values. A function which is a candidate for being the two-sided Laplace transform of a function $\psi(\mathbf{a})$ of connections will be denoted by a ${ }^{*}$-subscript $\psi_{*}(\mathbf{s})$. In addition, we will denote by $\tilde{\psi}$ the Fourier transform of $\psi(\mathbf{a})$ so that $\tilde{\psi}(x)=\psi_{*}(i x)$. A hat $(\hat{\psi})$ will denote the potential image of a Laplace transform evaluated at integer arguments; $\hat{\psi}(n)=\psi_{*}(n)$ for $n \in \mathcal{Z}$.

In trying to resolve our dilemma, we might first ask if the image of the loop transform $(\operatorname{Im}(\mathbf{L}))$ in the spacelike sector contains the "standard" loop representation (the set of $l^{2}$ functions loops) at all. For, if the loop transform defines a distinct loop representation in the spacelike sector by producing a representation containing no function with support on a single loop, then the proof in Appendix D would not apply and our dilemma would be resolved. Are any such functions of finite support in $\operatorname{Im}(\mathbf{L})$ ? In fact, they all are, and we can use the above theorem to show this.

Note that the function

$$
\psi_{* n}(s)=e^{\alpha s^{2}}\left(\frac{\sin \pi(s-n)}{\pi(s-n)}\right)^{2}
$$

takes on the values

$$
\hat{\psi}_{n}(m)=\delta_{n, m}
$$

at integer points. Also note that this function satisfies the conditions of our transform inversion theorem for any $\alpha \in \mathcal{R}^{+}$and so is the two-sided Laplace transform of some function $\psi(a)$. Since the Fourier transform preserves the $\mathcal{L}^{2}$ norm, we can use $\psi_{* n}(i x)=\tilde{\psi}(x)$ to evaluate $\|\psi\|_{\mathcal{L}^{2}}$ and find that $\psi \in \mathcal{L}^{2}(\mathcal{R})$. It follows that if we define $\Psi_{\mathbf{n}}(\mathbf{a}) \equiv \psi_{n_{1}}\left(a_{a}\right) \psi_{n_{2}}\left(a_{2}\right)+\psi_{n_{1}}\left(-a_{1}\right) \psi_{n_{2}}\left(-a_{2}\right)$, then $\Psi_{\mathbf{n}}$ is in $\mathcal{L}_{+}^{2}$ and

$$
\hat{\Psi}_{\mathbf{n}}(\mathbf{m})=\delta_{\mathbf{n}, \mathbf{m}}+\delta_{\mathbf{n},-\mathbf{m}}
$$

and $\hat{\Psi}_{\mathbf{n}}$ is nonzero on exactly one distinct loop.

Since the loop transform is a linear operator and its image contains the functions $\hat{\Psi}_{\mathbf{n}}$, all functions with support on a finite set of loops are also in the image of the loop transform. The image of the loop transform therefore contains at least a dense subset (in the $l^{2}$ topology) of the standard loop representation. A more subtle use of the

\footnotetext{
${ }^{6}$ We will refer to the set of such symmetric $\mathcal{L}^{2}$ functions as $\mathcal{L}_{+}^{2}$.
} 
transform inversion theorem shows that every $l^{2}$ function of loops is in the image of the loop transform. This fact is a straightforward consequence of the main proof given in Appendix A and so will be established at the end of this appendix. Of course, the transform inversion theorem can also be used to show that $\operatorname{Im}(\mathbf{L})$ contains functions of loops that are not in $l^{2}$ at all, but this only complicates our dilemma.

Instead of asking questions about the image of the loop transform we might ask questions about this transform's kernel. A nontrivial kernel would tell us that the loop transform is not a representation isomorphism in the spacelike sector and would go a long way toward resolving our dilemma. We can again use the transform inversion theorem to construct functions that are in the kernel of the loop transform. For example, consider the function:

$$
\psi_{*}(s)=\sin ^{2}(\pi s) e^{\alpha s^{2}}
$$

for $\alpha>0$. Note that this function satisfies the conditions of the transform inversion theorem and therefore is the the two-sided Laplace transform of some $\psi(a)$. Since the $\mathcal{L}^{2}$ norm is preserved by the Fourier transform, we see from Eq. 4.3 that $\psi \in \mathcal{L}^{2}(\mathcal{R})$ and therefore that the function $\boldsymbol{\Psi}(\mathbf{a})=\psi\left(a_{1}\right) \psi\left(a_{2}\right)$ is in the Hilbert space of the connection representation although its loop transform is the zero function: $(\mathbf{L} \Psi)\left(n_{1}, n_{2}\right)=\psi_{*}\left(n_{1}\right) \psi_{*}\left(n_{2}\right)=0$ for $\mathbf{n} \in \mathcal{Z}^{2}$ ? We conclude that $\mathbf{L}$ has a nontrivial kernel; it is not a representation isomorphism.

Let us now try to answer the question "How bad is it?" In other words, how big is the kernel of the loop transform and just what is the relationship between the connection and loop representations in the spacelike sector? A brief look at the discussion above indicates that the kernel $K(\mathbf{L})$ of $\mathbf{L}$ may be large indeed since $\hat{\psi}$ samples only the integer values of $\psi_{*}$. However, not all functions $\psi_{*}$ are the Laplace transform of some $\psi \in \mathcal{L}^{2}$ so this argument cannot be naively used to describe $K(\mathbf{L})$. In particular, if $\hat{\psi}(n)=\mathbf{L} \psi(n)$ is defined for all $n \in \mathcal{Z}$, then the integral defining the Laplace transform converges for all complex $n$ and does so uniformly on each compact subset of the complex plane. Because this integral converges uniformly in any compact region, we can differentiate the resulting function with respect to $n$ by interchanging the orders of integration and differentiation. Therefore, since $e^{a n}$ is analytic in $n$ for all complex $n, \psi_{*}(s)$ satisfies the Cauchy-Riemann equations and is also analytic for all complex $s$. This restriction that $\psi_{*}$ be analytic is a rather strong one and could severly restrict the functions in the kernel, making the kernel much smaller than we might have anticipated. In addition to this, there is still the restriction that $\psi(a)$ be normalizable in $\mathcal{L}^{2}$. A more careful examination will be required to determine the status of this kernel.

The most benign possibility is that $K(\mathbf{L})$ is finite dimensional. In this case, we could reasonably conclude that the loop representation contains "almost all" of the information in the connection representation and attempt to supplement the loop representation with an additional (discrete) degree of freedom. However, this loop representation would still contain all functions with support on a finite number of loops (a dense set in the standard representation) and, if the discrete label does indeed remove the ambiguities associated with the loop functions then the loop function that is always zero would again represent the zero state, at least for some value of the discrete index. This loop representation must again be a topologically irreducible representation of the $\mathcal{T}^{0}$ 's and $\mathcal{T}^{1}$ 's since $\mathcal{L}_{+}^{2}$ is and it follows that the comments in Appendix D still show that the loop representation and its inner product are uniquely given by the standard loop representation. Thus, the loop representation would still have to be identical to the standard loop representation and our dilemma would remain unresolved.

However, this is not the case. If the function given in 4.7 is multiplied by any polynomial in $s$, it still satisfies the conditions of the transform inversion theorem and still gives zero when evaluated at any integer. Since these functions are linearly independent, $K(\mathbf{L})$ must be infinite dimensional.

The next most benign possibility is that $K(\mathbf{L})$ is closed as a subspace of $\mathcal{L}_{+}^{2}$. In this case we might still argue that the loop representation captures an essential part of the connection representation. Then, the connection representation $\mathcal{A}$ would be related to the loop representation, $L$, by $\mathcal{A} \cong K(\mathbf{L}) \oplus \mathcal{A} / K(\mathbf{L})$ and $\mathcal{A} / K(\mathbf{L}) \cong L$ so that $\mathcal{A} \cong K(\mathbf{L}) \oplus L$. Again, this loop representation must be just the standard loop representation. However, it turns out that the kernel is not in fact closed. The proof of this makes further use of the transform inversion theorem and is given in Appendix A. This appendix shows that the product of any two functions $\psi_{n}$ defined in 4.4 is in the closure of $K(\mathbf{L})$ and therefore that $K(\mathbf{L})$ is not closed.

A slight modification of this last proposal would be to make the decomposition $\mathcal{A} \cong \overline{K(\mathbf{L})} \oplus \mathcal{A} / \overline{K(\mathbf{L})}$, where the overbar represents the closure as a subspace of $\mathcal{L}_{+}^{2}$. However, $L \cong \mathcal{A} / \overline{K(\mathbf{L})}$ may be nothing like the standard loop representation since it might be that for some $\hat{\phi} \in \operatorname{Im} \mathbf{L}$, the elements of the connection representation that map to $\hat{\psi}$ via the loop transform are all contained in the closure of $K(\mathbf{L})$. In this case, even though $\hat{\phi} \in \operatorname{Im}(\mathbf{L})$, we would still find that $\phi \equiv 0$ in $A \overline{K(\mathbf{L})}$.

\footnotetext{
${ }^{7}$ Again, thanks to Jorma Louko for pointing out these constructions
} 
An important question is "Which $\hat{\phi}$ are identified with the zero state by this procedure?" Or, "How much of the connection representation does this loop representation capture?" These questions are related to the question, "How large is the kernel?" which is explored in Appendix A. By using the transform inversion theorem several times, this appendix shows that the kernel is so large that for any symmetric or antisymmetric function $\Lambda$ in $\mathcal{L}^{2}(\mathcal{R})$ of compact support, there is a sequence of $\mathcal{L}^{2}(\mathcal{R})$ functions that converges to $\Lambda$ in the $\mathcal{L}^{2}(\mathcal{R})$ topology such that the Laplace transforms of each function in this sequence vanishes for all integer values. Thus, the closure in this topology of the set of $\mathcal{L}^{2}(\mathcal{R})$ functions whose Laplace transforms vanish at all integer values contains all symmetric or antisymmetric $\mathcal{L}^{2}$ functions of compact support on the real line. Since this closure is closed, it must also contain the closure of the set of all symmetric and antisymmetric $\mathcal{L}^{2}$ functions of compact support, which is just the space of all symmetric and antisymmetric $\mathcal{L}^{2}$ functions. A dense subset of $\mathcal{L}_{+}^{2}$ consists of a sum of products of symmetric $\mathcal{L}^{2}(\mathcal{R})$ functions in each variable and products of antisymmetric $\mathcal{L}^{2}(\mathcal{R})$ functions in each variable. It follows that $K(\mathbf{L})$ is dense in $\mathcal{L}_{+}^{2}$ and that $\mathcal{L}_{+}^{2} / \overline{K(\mathbf{L})} \cong\{0\}$ ! Thus there is a sense in which the loop transform does not capture any part of the connection representation.

This result is indeed strong enough to let us out of our dilemma. If we look simply at the image $\operatorname{Im}(\mathbf{L})$ as a loop representation then the zero function does not necessarily represent the zero state. If we look at the quotient $\operatorname{Im}\left(\mathcal{L}^{2}\right) / \overline{K(\mathbf{L})}$ then this space does not contain any function of loops that is nonzerop on exactly one loops. In fact, it doesn't contain any non-zero functions of loops at all. Neither choice satisfies the conditions required in Appendix D so that the proof given there has nothing at all to say about the character of the resulting loop representation.

\section{CONSTRUCTING A "LOOP" REPRESENTATION}

We have seen that there is a sense in which the loop transform does not capture any part of the connection representation. Is there another sense in which we can construct a "loop representation" that is in fact isomorphic to the connection representation? Despite the discussion of the last section, we shall see that the answer to this question is, "Yes.". We begin by recalling that a loop representation is a representation of the $\mathcal{T}^{0}, \mathcal{T}^{1}$ algebra on some space which is the completion of some linear space of functions of loops. This means that we do not need to map every element of the Hilbert space of the connection representation to a function of loops, but only the elements of some dense subset, $\mathcal{V}$.

The idea is to construct a loop representation by carefully choosing this dense subset. While $K(\mathbf{L})$ is dense in $\mathcal{L}_{+}^{2}$, suppose that it is also true that there exists some dense, linear subspace, $\mathcal{V} \subset \mathcal{D}_{\mathbf{L}}$ and such that $\mathcal{V} \cap K(\mathbf{L})=\{0\}$. Let us also assume that $\mathcal{V}$ is stable under the $\mathcal{T}^{0}$ 's and $\mathcal{T}^{1}$ 's so that it does, in fact, provide a representation of our basic algebra. Since $\mathcal{V} \cap K(\mathbf{L})=\{0\}$, it follows that $\mathcal{V}$ and $\mathbf{L}(\mathcal{V})$ are isomorphic as representations of the $\mathcal{T}^{0}$ 's and $\mathcal{T}^{1}$ 's. If we now define the inner product on $\mathbf{L}(\mathcal{V})$ by $\langle\mathbf{L} \phi, \mathbf{L} \psi\rangle \equiv\langle\phi, \psi\rangle$, then $\mathcal{V}$ and $\mathbf{L}(\mathcal{V})$ are isomorphic as vector spaces with an inner product. Both of these spaces may be completed in the topologies defined by their inner products and the results must be isomorphic as Hilbert spaces.

This Hilbert space isomorphism can now be used to define the action of the $\mathcal{T}^{0}$ 's and $\mathcal{T}^{1}$ 's in the completion of $\mathbf{L}(\mathcal{V})$ in such a way that this completion $\overline{\mathbf{L}(\mathcal{V})}$ and the completion of $\mathcal{V}$ are isomorphic as representations of the $\mathcal{T}^{0}, \mathcal{T}^{1}$ algebra. But, as the completion of $\mathcal{V}$ is $\mathcal{L}_{+}^{2}$, our "loop representation" 9 is isomorphic to $\mathcal{L}_{+}^{2}$ as a Hilbert space representation of the $\mathcal{T}^{0}, \mathcal{T}^{1}$ algebra. If such a $\mathcal{V}$ exists, we see that it defines a loop representation which is isomorphic to the connection representation, although we had to take special care to construct it.

The construction of such subspaces and the proof that they satisfy our requirements requires additional care, so we carry it out in Appendix B. In fact, in Appendix B we show that there are two such subspaces. Note that the preceding paragraph shows that the representation constructed from either subspace is isomorphic to the connection representation and therefore that these two representations are isomorphic to each other. Therefore, the prescription given above defines a unique loop representation up to representation isomorphism.

Despite the uniqueness of this loop representation in the sense just mentioned, there are still ambiguities in the formulation of these representations in terms of loops. We should note that the above procedure constructs loop representations only in a technical sense. That is, they are completions of a linear space of functions of loops, but may therefore contain many elements that cannot, in fact be described as a function of loops.

We should also be aware that even though these loop representations are isomorphic, this does not mean that the isomorphism acts in a way that preserves the parts of these representations which are formed by functions of loops.

\footnotetext{
${ }^{8}$ Recall that two dense subspaces can have trivial intersection.

${ }^{9}$ The closure of $\mathbf{L}(\mathcal{V})$ carries a representation of the loop algebra and therefore defines a loop representation.
} 
Nor does it mean that functions of loops have a well defined correspondence with functions of connections without reference to the specific subspace $\mathcal{V}$ used to construct that loop representation. We shall see that a given function of loops may correspond to a different function of connections depending on which subspace was chosen. These functions of connections may even have different norms so that the inner product on functions of loops also depends on the subspace $\mathcal{V}$ that was chosen. In fact, which functions of connections correspond to functions of loops at all will also depend on the choice of a dense subspace $\mathcal{V}$.

To see why these ambiguities arise, let's try the above construction with two subspaces, $\mathcal{V}_{1}, \mathcal{V}_{2}$, both satisfying the conditions given above. First, we notice that for any $\hat{\phi} \in \operatorname{Im}(\mathbf{L})$, there is a dense subspace of the carrier space of the connection representation that maps onto $\hat{\phi}$. Since only one of these can be represented by $\hat{\phi}$, only a rather small part of this carrier space will correspond to functions of loops. Just which $\phi$ will be so represented depends on the choice of $\mathcal{V}$; if there is some $\phi_{1} \in \mathcal{V}_{1}$ and some $\phi_{2} \in \mathcal{V}_{2}$ such that $\mathbf{L} \phi_{1}=\hat{\phi}=\mathbf{L} \phi_{2}, \hat{\phi}$ would represent $\phi_{1}$ in the representation constructed from $\mathcal{V}_{1}$ and would represent $\phi_{2}$ in the representation constructed from $\mathcal{V}_{2}$. Since the function $\hat{\phi}$ already represents $\phi_{1}$ in the first representation there is no way for $\phi_{2}$ to be represented there by a function of loops consistent with the loop transform. If $\left\|\phi_{1}\right\| \neq\left\|\phi_{2}\right\|$, then $\|\hat{\phi}\|$ also depends on which $\mathcal{V}$ we used as a starting point and therefore so does the form of the inner product in terms of functions of loops. We show in Appendix $B$ that $\mathcal{V}_{1}$ and $\mathcal{V}_{2}$ as discussed above do indeed exist. Therefore, we cannot hope that the requirements imposed on $\mathcal{V}$ conspire to make the choice of $\mathcal{V}$ unique or to remove the above ambiguities.

However, all known subspaces $\mathcal{V}$ that satisfy the above requirements are highly algebraically reducible representations of the $\mathcal{T}^{0}$ 's and $\mathcal{T}^{1}$ 's. They have the property that every linear subrepresentation of the $\mathcal{T}^{0}$ 's and $\mathcal{T}^{1}$ 's is also algebraically reducible. It would be fairly natural to require the subspace $\mathcal{V}$ that we will use to construct a loop representation to be an algebraically irreducible representation of the $\mathcal{T}^{0}$ 's and $\mathcal{T}^{1}$ 's and it might be hoped that such a requirement would select a unique subspace or at least remove the above mentioned ambiguities in the loop representation. At present, it is not known if any such subspaces of $\mathcal{L}_{+}^{2}$ exist. If they do, we could then explore the question of uniqueness.

\section{GENERALIZED LOOP COORDINATES AND THE INNER PRODUCT}

While we have been unable to remove these ambiguities in our loop representation, there $i s$ a way to construct a related but different representation that is not subject to such ambiguites. This representation will not be formulated in terms of functions of loops, but in terms of functions of "generalized" loops. Before we construct this representation, we will briefly review the definitions of generalized loops and the corresponding generalized holonomies from [24.26] and a few of the relevant facts derived in those papers. We will then proceed to construct a generalized loop representation using suitable functions of generalized loops and generalized loop analogues of the $\mathcal{T}^{0}$ 's and $\mathcal{T}^{1}$ operators. This construction will again rely heavily on a transform which is a generalized version of the loop transform.

We now abandon the practice of sections III-V of using "loops" to mean equitopy classes of loops. In this section "loop" will refer to an equivalence class under reparameterization of smooth maps from $[0,1]$ to some two manifold $\Sigma$ that map both 0 and 1 to some fixed base point, ${ }^{*}$, in the manifold. We also identify two such maps $\alpha$ and $\beta$ if the composition of based loops $\alpha \circ \beta^{-1}$, where $\beta^{-1}$ is the loop $\beta$ followed in the reversed direction, is contractible within itself to the trivial loop. These equivalence classes of based loops will be the starting point for our description of generalized loops.

The idea behind generalized loops, also called extended loops and loop coordinates, is to generalize loops in such a way as to form a space that has the local structure of a Lie group [24 25]. Note that while the space of loops as defined above has a natural group structure in which multiplication of two loops is given by the composition of pointed loops at their base point, this is not a Lie group structure since there are no one-parameter subgroups. For example, if $\alpha$ is injective as a map into its image then there is no loop that gives $\alpha$ when squared. Roughly speaking then, the "extended group of loops" contains elements that "fill in" between the zero loop and once around a given loop. It also contains elements that fill in between once around a given loop and twice around that loop. Suppose that we label this one-parameter subgroup of extended loops $l_{\lambda}$ by a real number $\lambda$. For integer $\lambda, l_{\lambda}$ is the set of loop coordinates of the loop that wraps $\lambda$ times around our given loop and for all real $\lambda_{1}$ and $\lambda_{2}$ these labels satisfy $l_{\lambda_{1}} \times l_{\lambda_{2}}=l_{\lambda_{1}+\lambda_{2}}$, where $\times$ is the group multiplication. We might therefore say that these extended loops correspond to a "loop" that wraps $\lambda$ times around our given loop even for real $\lambda$. We might also guess that the extension in this sense of our set of equitopy classes of loops $\mathcal{Z}^{2} / \mathcal{Z}_{2}$ on the torus is just $\mathcal{R}^{2}$ or perhaps $\mathcal{R}^{2} / \mathcal{Z}_{2}$. This is in fact the correct result, though we will need to introduce some of the formalism of [24,26] to show this explicitly.

For convenience, we will not introduce the full formalism of loop coordinates since it is unnecessary for our simple model. In particular, we will only introduce enouugh formalism to describe generalized loops for flat abelian connections. This will suffice for our purposed since, in the $\epsilon_{1}=+1=\epsilon_{2}$ spacelike sector, every flat connection on the torus 
is gauge equivalent under small gauge transformations to some homogeneous connection. This is easy to check since the $S U(1,1)$ elements $\exp \left[A_{b}^{I} \tau_{I}\right]$ for two parallel $2+1$ dimensional internal Minkowsi vectors $\mathbf{A}_{\mathbf{b}}$ are the holonomies corresponding to a single loop in the $x_{b}$ direction given by the homogeneous connection whose components are just this same set of numbers $A_{b}^{I}$ at each point on the torus. Here we are using a standard set of coordinates $\left(x^{1}, x^{2}\right)$ on the torus with unit period. Such a connection behaves much like an abelian connection since the connection evaluated at any point on the torus and contracted with some tangent vector to the torus at that point commutes with the connection evaluated at any other point and contracted with any tangent vector at this second point.

The holonomies of an abelian connection are simply exponentials of integrals over each loop:

$$
\begin{aligned}
U[\alpha](A) & =\exp \left(\int_{\alpha} d x^{a} A_{a}^{I} \tau_{I}\right) \\
& =\exp \left(\int d^{2} x A_{a}^{I} \tau_{I}\left(\int_{0}^{1} d s \dot{\alpha}^{a}(s) \delta^{2}(\alpha(s)-x)\right)\right)
\end{aligned}
$$

The distribution: $\int_{0}^{1} d s \dot{\alpha}^{a}(s) \delta^{2}(\alpha(s)-x) \equiv T_{\alpha}^{a}(x)$ forms the set of so-called "loop coordinates" for the loop $\alpha$. Note that this distribution satisfies:

$$
\partial_{a} T_{\alpha}^{a}(x)=0
$$

for any loop $\alpha$. Note that $T_{n \alpha}^{a}(x)=n T_{\alpha}^{a}(x)$. Thus, one way to "fill in" the gaps between the loops is to consider any "form factor" that satisfies Eq. 6.2 to define a generalized loop.

Corresponding to any such generalized loop, we can define a "generalized holonomy" by

$$
U_{T}(A)=\exp \left(\int d^{x} T^{a}(x) A_{a}^{I}(x) \tau_{I}\right)
$$

Note that the condition (6.2) on the form factors guarrantees gauge invariance of the holonomies (since the connections are abelian). Juust as for loops, we will place the generalized loops into equivalence classes so that two generalized loops are equivalent if they give the traces of their generalized holonomies are the same for every connection $A$ in whatever class of connections we are considering.

Let us now compute the generalized holonomies that correspond to the form factors that satisfy Eq. 6.2. Note that this equation implies that $T^{a}(x)$ must be of the form: $T^{a}(x)=\epsilon^{a b} \partial_{b} \Lambda(x)+s^{a}$ for some function $\Lambda$ and some constant vector $s^{a}$. We can use our homogeneous connections to calculate these generalized holonomies so that:

$$
\begin{aligned}
U_{T}(A) & =\exp \left[\int_{\mathcal{T}^{2}} d^{2} x A_{b}^{I}(x) T^{b}(x) \tau_{I}\right] \\
& =\exp \left[\int_{\mathcal{T}^{2}} d^{2} x\left(\epsilon^{b c} \partial_{c} \Lambda A_{b}^{I}\right) \tau_{I}+A_{b}^{I} \tau_{I} s^{b}\right]=\exp \left(A_{b}^{I} s^{b} \tau_{I}\right)
\end{aligned}
$$

and distinct equivalence classes of generalized loops depend only on the vector $\mathbf{s}=\left(s^{1}, s^{2}\right)$.

Because these generalized holonomies are boosts, each choice of $\mathbf{s} \in \mathcal{R}^{2}$ leads to a distinct set of generalized holonomies. However, $\mathbf{s}$ and $-\mathbf{s}$ provide the same set of traces. Thus, we make one last identification of $\mathbf{s}$ with $-\mathbf{s}$ and find that our equivalence classes are labeled exactly by $\mathbf{s} \in \mathcal{R}^{2} / \mathcal{Z}_{2}$, just as we expected.

To complete our generalized loop representation, we would like to form $\mathcal{T}^{0}$ 's and $\mathcal{T}^{1}$ 's for each equivalence class $\mathbf{s}$ of generalized loops. This can be done by simply replacing every holonomy in the definitions of these functions given in Eq. 2.10 with a generalized holonomy. Note that the $\mathcal{T}^{1}$ 's are again well-defined as functions of our equivalence classes of generalized loops.

These functions of generalized loops also satisfy the Poisson Bracket relations given in Eq. 2.11 and the algebraic relations given in Eq. 2.12 although the generalized loop indices are now allowed to be real. These functions can be expressed explicitly in the connection representation as:

$$
\begin{gathered}
\mathcal{T}^{0}[\mathbf{s}](\mathbf{a})=\cosh (\mathbf{s} \cdot \mathbf{a}) \\
\mathcal{T}^{1}[\mathbf{s}](\mathbf{a})=\sinh (\mathbf{s} \cdot \mathbf{a}) \mathbf{s} \times \mathbf{p}
\end{gathered}
$$

and in the generalized loop representation as:

$$
\left(\mathcal{T}^{0}(\mathbf{t}) \psi\right)(\mathbf{s})=(\psi(\mathbf{s}+\mathbf{t})+\psi(\mathbf{s}-\mathbf{t})) / 2
$$




$$
\left(\mathcal{T}^{1}(\mathbf{t}) \psi\right)(\mathbf{s})=i \mathbf{t} \times \mathbf{s}(\psi(\mathbf{s}+\mathbf{t})-\psi(\mathbf{s}-\mathbf{t}))
$$

We can also define a generalized loop transform by integrating any function in the connection representation against traces of the generalized holonomies. Thus, we evaluate the loop transform integral not just for traces of holonomies around loops, but also for traces of generalized holonomies of generalized loops. From Eq. 6.5a, we see that the loop transform is again the two-sided Laplace transform, but it is now evaluated at every $\mathbf{s} \in \mathcal{R}^{2}$. Note that if the Laplace transform exists for some $s_{1}, s_{2} \in \mathcal{R}$, then it exists for all $s_{1}<s<s_{2}$ and that therefore the existence of the generalized loop transform for all real $s$ is equivalent to the existence of the loop transform for all integer $n$ and the domain of this generalized loop transform in the connection representation is the same as the domain $\mathcal{D}_{\mathbf{L}}$ of the original loop transform.

The generalized loop representation avoids the ambiguities that we found for the original loop representation because it has trivial kernel. To see this, recall that if the Loop transform integral converges for all real $\mathbf{s}$, then $\psi_{*}(\mathbf{s})=\int d^{2} a e^{\mathbf{s} \cdot \mathbf{a}} \psi(\mathbf{a})$ for all complex $\mathbf{s}$. But, if $\psi \in K(\mathbf{L}), \psi_{*}(\mathbf{s})=0$ for all complex $\mathbf{s}$ and $\tilde{\psi}(\mathbf{x})=0$ as well. But $\tilde{\psi}(\mathbf{x})$ is just the Fourier transform of $\psi(\mathbf{a})$ and the Fourier transform has trivial kernel in $\mathcal{L}_{+}^{2}$. It follows that $\psi(\mathbf{a})=0$ and that the kernel of the generalized loop transform is trivial.

This delightful fact means that we may construct our generalized loop representation in much the same way that we built our loop representations in section $\mathrm{V}$ but that we may now use all of $\mathcal{D}_{\mathbf{L}}$ as the dense subspace whose image we complete to define the Hilbert space. As before, we define the inner product in the image of $\mathcal{D}_{\mathbf{L}}$ to be given by the inner product in $\mathcal{D}_{\mathbf{L}}$ and complete the resulting space with respect to this inner product. This produces an unambiguous representation in terms of (generalized) functions of generalized loops that is isomorphic to the connection representation. We must consider suitably generalized functions since the generalized loop transform is still not defined for all $\psi \in \mathcal{L}_{+}^{2}$ and the completion of $\operatorname{Im}\left(\mathcal{D}_{\mathbf{L}}\right)$ will contain elements that are not functions of generalized loops. The representation is unambiguous in the sense that the part of the representation that can be described by functions of loops is exactly the same as what would have been obtained if any dense subset $\mathcal{V}$ of $\mathcal{D}_{\mathbf{L}}$ were used in the construction instead of $\mathcal{D}_{\mathbf{L}}$ itself.

Similarly, we conclude that a given function of generalized loops (in the image of $\mathcal{D}_{\mathbf{L}}$ ) has a unique norm and a unique inner product with any other such function of generalized loops. We can even find an explicit form for this inner product using our transform inversion theorem. Remembering that our functions in the image of $\mathcal{D}_{\mathbf{L}}$ are holomorphic on the complex two-plane $\mathcal{C}^{2}$, they have a unique and well defined analytic continuation to the imaginary axes. The inner product is given in terms of this analytic continuation by:

$$
\langle\phi, \psi\rangle=\int d^{2} x\left(\phi_{*}(i \mathbf{x})\right)^{\star} \psi_{*}(i \mathbf{x})
$$

where $(\star)$ represents complex conjugation and $(*)$ as usual denotes a function of generalized loops.

The completion of the image of $\mathcal{D}_{\mathbf{L}}$ can be described easily in terms of functions of the imaginary coordinate $\mathbf{x}=i \mathbf{s}$ as the $\mathcal{L}_{+}^{2}\left(\mathcal{R}^{2}\right)$ space. However, an attempt to describe this space completely in terms of functions of generalized loops (the real axis) involves a prescription for analytic continuation of an arbitrary $\mathcal{L}^{2}\left(\mathcal{R}^{2}\right)$ function defined along the imaginary axes to the real axes. Some sort of additional structure would have to be introduced for this purpose.

On the other hand, suppose for a moment that we wish to change our position and think about functions of "imaginary generalized loops" (i.e., to work with functions defined along the imaginary axis). While this provides a representation that is clearly equivalent to the original connection representation and in which it is easy to compute inner products, the action of the $\mathcal{T}^{0}$ 's and $\mathcal{T}^{1}$ 's in this representation is far from simple. The $\mathcal{T}^{0}$ 's and $\mathcal{T}^{1}$ 's are then represented by non-local differential operators, although the original $\mathbf{a}$ and $\mathbf{p}$ are represented simply.

We therefore see that the use of generalized loops eliminates all of the ambiguities that we found in section $\mathrm{V}$. However, it is still true that not all states in the generalized loop representation can be regarded as functions even of generalized loops. Interestingly, they can be regarded as functions of "imaginary generalized loops" and the inner product can be expressed conveniently using this description, though this is at the cost of loosing the simple form of the $\mathcal{T}^{0}$ 's and $\mathcal{T}^{1}$ operators. In the end, this representation in terms of imaginary generalized loops is just the momentum representation obtained by Fourier transform of the connection representation.

\section{DISCUSSION}

In thinking about this loop transform, one analogy has proved itself to be extremely useful. This is an analogy with the so-called (integer) "Mellin transform,"

$$
\psi_{*}(n) \equiv \int_{-\infty}^{\infty} x^{n} \psi(x) d x
$$


for $\psi \in \mathcal{L}^{2}(\mathcal{R})$. Here, the multiplication operator $x^{n}$ plays the role of the $\mathcal{T}^{0}(\mathbf{k})$ 's and the differential operators $-i x^{n} \frac{\partial}{\partial x}$ play the part of the $\mathcal{T}^{1}(\mathbf{k})$ 's. These operators have a commutator algebra reminiscent of the loop algebra and the set of polynomials in $x$ provides a cyclic representation for these operators.

If we consider the action of the Mellin transform in the momentum representation:

$$
\psi(n)=\int x^{n} \tilde{\psi}(p) e^{-i x p} d x d p=\left.2 \pi\left(-i \frac{\partial}{\partial p}\right)^{n} \tilde{\psi}(p)\right|_{p=0}
$$

we see that the resulting function of integers determines $\psi$ uniquely only if we require that $\tilde{\psi}(p)$ be analytic. All functions of momenta that are zero in some neighborhood of the origin are in the kernel of this transform and this set is dense in $\mathcal{L}^{2}(\mathcal{R})$. Again, several dense linear subspaces of $\mathcal{L}^{2}$ can be found that provide a representation of the above algebra but don't intersect the kernel of the transform and again the images of these subspaces overlap. This analogy may continue to be useful in future study of the loop transform.

Despite the dense kernel of the loop transform, we did find that a loop representation can be constructed in the spacelike sector that is isomorphic to the connection representation. The construction of this loop representation is based on the choice of some dense subspace $\mathcal{V}$ of the connection representation satisfying certain conditions. Because the construction process guarantees that the loop representation is isomorphic to the connection representation, the loop representation constructed using any choice of suitable dense subspace is isomorphic to that constructed using any other such choice. However, a number of ambiguities remain concerning just how this representation is described in terms of loops and it is difficult to see how the loops themselves could be considered to be the fundamental objects in the theory. It is possible that requiring the dense subspace space $\mathcal{V}$ to be an algebraically irreducible representation of the $\mathcal{T}^{0}$ 's and $\mathcal{T}^{1}$ 's will remove these ambiguities. However, at present it is not even known if such a subspace exists.

While we have confined ourselves to $2+1$ gravity on a torus, we might try to use our results to draw more general conclusions. There are, however, several potential obstacles to doing so. One potential obstacle is that $2+1$ dimensional gravity on a torus is an abelian theory, in contrast to $3+1$ gravity or even $2+1$ gravity on higher genus spatial slices. Another potential obstacle is that we have not considered the possibility of imposing identifications in the phase space under large diffeomorphisms. To impose such identifications, we would have to face two problems: first, that the large diffeomorphisms do not act properly discontinuously on our reduced phase space; second, that the $\mathcal{T}^{0}$ 's and $\mathcal{T}^{1}$ 's are not invariant under large diffeomorphisms. This second problem means that special consideration would be required to define the loop transform if we quotient the reduced phase space by the large diffeomorphisms.

Despite these potential obstacles, let us briefly speculate about $2+1$ dimensional gravity on $\Sigma \times \mathcal{R}$, where $\Sigma$ is a compact surface with genus greater than one. Note that the reduced configuration space (without dividing by the large diffeomorphisms) has infinite volume 10 and that the holonomies are boosts in the component of the phase space that corresponds to classical spacetimes with no closed timelike curves [23]. Because of this, the $\mathcal{T}^{0}(\mathbf{k})$ 's are unbounded and we would expect results similar to the spacelike sector of the torus. It should be emphasized, however, that at this point this is only speculation by analogy.

We might also try to draw conclusions about loop representations in $3+1$ dimensional gravity. However, the $3+1$ theory is extremely different, not least because the $\mathcal{T}^{0}$ 's and $\mathcal{T}^{1}$ 's are no longer classically real and so cannot be taken to be Hermitian in the quantum theory. This means that the inner product must be determined in a different way in the $3+1$ theory. Since it is the inner product that determines the continuity of the loop transform, this makes it unclear what implications our work holds for the $3+1$ theory.

However, when trying to use our results to discuss the $3+1$ theory, the fact that we did not take the quotient of the reduced phase space by the large diffeomorphisms is actually not an obstacle, but an advantage. $2+1$ gravity on a torus is a theory with a finite number of degrees of freedom (2), describing only the global modes of a $2+1$ spacetime and the rearrangement of our set of loops under large diffeomorphisms is a direct consequence of their global nature and the fact that they represent homotopy classes. On the other hand, "loops" in the $3+1$ theory represent not homotopy classes of loops, but much smaller classes of loops that only contain loops that differ from each other by retracings 16. This is related to the fact that $3+1$ gravity is a field theory with both local and global degrees of freedom. Given two independent holonomies (loops) in the $3+1$ theory, there is no diffeomorphism (large or small) that maps these loops to products of themselves as the large diffeomorphisms map the loops in the $2+1$ theory. We may therefore hope that we have chosen a description of $2+1$ gravity that can teach us about the $3+1$ theory.

One general lesson that we can claim to have learned here is to be wary of formal arguments using the loop transform. In general, extra structure may be required in order to give the loop representation the desired properties.

\footnotetext{
${ }^{10}$ This follows from a result in 30]. Thanks to Steve Carlip for help finding this reference.
} 
The resulting loop representation may be nothing like what might have been expected by thinking only about loops. Indeed, if the goal is equivalence between the connection and loop representations - or, more generally, to ensure that the loop representation provides the correct classical limit - then it is difficult to see what conceptual role the loops themselves take on.

On the other hand, generalized loops brought us much more success. Though the inner product on the space of functions of generalized loops is still quite complicated, at least it is well defined. Because generalized loops form a vector space and lack the discrete structure of loops, they are easier to link to a connection representation. However, this same feature can make them less appealing to those who would like to see a discrete structure for spacetime. Constructions analogous to the weave states and to various operators that have been defined in loop representations [8,7]20] may have to be modified and the conclusions rethought if they are reformulated in terms of generalized loops. Yet, it seems likely that generalized loops will continue to play a significant role in the study of loop representations.

On the whole, we have seen that the construction of a loop representation in the spacelike sector of $2+1$ quantum gravity is a subtle issue. This is a consequence of the fact that the loop transform in the spacelike sector is not everywhere defined on the connection Hilbert space fails to be continuous with respect to the natural topologies, even in its domain of definition. Any attempt to define the loop representation by completion of all or part of the image of the domain of definition of the loop transform must somehow introduce some extra structure to deal the the issues raised by lack of continuity.

\section{ACKNOWLEDGMENTS}

The author would like to express his thanks to Abhay Ashtekar and Jorma Louko for numerous questions and comments. Special thanks go to Abhay Ashtekar for editorial comments, to Alan Rendall for punctuating the difference between algebraic and topological reducibility, and to Jorma Louko for asking the question "Is the kernel dense?" This work was supported in part by the NSF grant PHY XXX to Syracuse University and by research funds provided by Syracuse University.

\section{APPENDIX A: THE KERNEL IS DENSE}

In order to show that the kernel $K(\mathbf{L})$ is dense in $\mathcal{L}_{+}^{2}$, we will show that for each continuous $\Lambda \in \mathcal{L}_{+}^{2}$ of compact support that is invariant under $\mathbf{a} \rightarrow-\mathbf{a} \mathbb{1}$ there is a sequence, $\left\{\phi^{\Lambda,(k)}\right\} \subset K(\mathbf{L})$ such that $\phi^{\Lambda,(k)} \rightarrow \Lambda$ in the $\mathcal{L}^{2}$ norm as $k \rightarrow \infty$. From this, it will follow that the closure $\overline{K(\mathbf{L})}$ of the kernel contains $\mathcal{C}_{0+}^{0}\left(\mathcal{R}^{2}\right)$ and that therefore we have $\overline{K(\mathbf{L})} \supset \overline{\mathcal{C}_{0+}^{0}\left(\mathcal{R}^{2}\right)} \supset \mathcal{L}_{+}^{2}$. We conclude that $K(\mathbf{L})$ is dense in $\mathcal{L}_{+}^{2}\left(\mathcal{R}^{2}\right)$.

The sequence, $\left\{\phi^{\Lambda,(k)}\right\}$, will be constructed by first finding a sequence of functions, $\left\{\psi^{\Lambda,(k)}\right\}$, that have the same loop components as $\Lambda$ :

$$
\hat{\psi}^{\Lambda,(k)}(\mathbf{n})=\hat{\Lambda}(\mathbf{n})
$$

This sequence will also have the property that $\left\{\psi^{\Lambda,(k)}\right\} \rightarrow 0$ as $k \rightarrow \infty$. This sequence will in turn be constructed from a sequence $\left\{\psi_{\mathbf{n}}^{(k)}\right\}$ of sets of "characteristic functions of loops" which have the properties that $\hat{\psi}_{\mathbf{n}}^{(k)}(\mathbf{m})=\delta_{\mathbf{n}, \mathbf{m}}{ }^{\text {[2 }}$ and that $\psi_{\mathbf{n}}^{k} \rightarrow 0$ in $\mathcal{L}_{+}^{2}$ as $k \rightarrow \infty$. We then define $\psi^{\Lambda,(k)} \equiv \sum_{m=0}^{\infty} \hat{\Lambda}(\mathbf{m}) \psi_{\mathbf{m}}^{(k)}$ so that $\hat{\psi}^{\Lambda,(k)}(\mathbf{n})=\hat{\Lambda}(\mathbf{n})$. The function $\phi^{\Lambda,(k)}$ is defined by $\phi^{\Lambda,(k)} \equiv \Lambda-\psi^{\Lambda,(k)}$. The proof consists of showing the existence of the functions $\psi_{\mathbf{n}}^{(k)}$ and that the above limits behave appropriately. Actually, we do all of this in the (completely) symmetric and antisymmetric parts $\left(\mathcal{L}_{++}^{2}\right.$ and $\left.\mathcal{L}_{--}^{2}\right)$ separately so that we can work with functions with one-dimensional arguments. The proof for our functions of two-dimensional arguments follows by taking tensor products of function spaces.

We begin by recalling that the loop transform is just the two sided Laplace transform in each variable. Therefore, if we can write our space of functions of connections in an appropriate way in terms of functions of one-dimensional arguments, we can work with the Laplace transform separately in each argument. To this end, we split the connection representation into completely symmetric and completely antisymmetric pieces, $\mathcal{L}_{+}^{2}=\mathcal{L}_{++}^{2} \oplus \mathcal{L}_{--}^{2}$. The space $\mathcal{L}_{++}^{2}$ consists of functions that are symmetric in $a_{1}$ and $a_{2}$ separately and the space $\mathcal{L}_{--}^{2}$ contains those functions that

\footnotetext{
${ }^{11}$ We will refer to this set of functions as $C_{0+}^{0}\left(\mathcal{R}^{2}\right)$

${ }^{12}$ The symbols $\mathbf{n}$ and $\mathbf{m}$ are taken to label equivalence classes of loops, i.e. $\mathbf{n}, \mathbf{m} \in \mathcal{R}^{2} / \mathcal{Z}^{2}$.
} 
are antisymmetric in both coordinates. A dense subset of $\mathcal{L}_{++}^{2}\left(\mathcal{L}_{--}^{2}\right)$ consists of sums of products of symmetric (antisymmetric) functions with one-dimensional arguments:

$$
\mathcal{L}_{++}^{2}\left(\mathcal{R}^{2}\right)=\mathcal{L}_{+}^{2}(\mathcal{R}) \otimes \mathcal{L}_{+}^{2}(\mathcal{R}) \text { and } \mathcal{L}_{++}^{2}\left(\mathcal{R}^{2}\right)=\mathcal{L}_{+}^{2}(\mathcal{R}) \otimes \mathcal{L}_{+}^{2}(\mathcal{R})
$$

where the \pm subscripts on $\mathcal{L}^{2}(\mathcal{R})$ again denote the symmetric and antisymmetric parts. Thus, if we can show that the kernel of the two-sided Laplace transform evaluated at integer points is dense in $\mathcal{L}_{+}^{2}(\mathcal{R})$ and $\mathcal{L}_{-}^{2}(\mathcal{R})$, it will follow that the kernel of the loop transform is dense in $\mathcal{L}_{+}^{2}\left(\mathcal{R}^{2}\right)$.

For convenience, we introduce the symbol $\mathbf{L}^{1}$ to denote this two-sided Laplace transform of a functions of a onedimensional argument; that is,

$$
\hat{\psi}(n)=\left[\mathbf{L}^{1} \psi\right](n)=\int_{-\infty}^{\infty} d a e^{n a} \psi(a)
$$

for all $n \in \mathcal{Z}$. We also define $K_{ \pm}\left(\mathbf{L}^{1}\right)$ and $\mathcal{D}_{ \pm \mathbf{L}^{1}}$ as subsets of $\mathcal{L}_{ \pm}^{2}(\mathcal{R})$ by analogy with $K(\mathbf{L})$ and $\mathcal{D}_{\mathbf{L}}$.

A fundamental part of our proof will be the "characteristic functions of loops" defined in Eq. 4.4. We now define the symmetrized and antisymmetrized combinations:

$$
\psi_{* n}^{ \pm(k)}(s)=\left(\psi_{* n}(s) \pm \psi_{*-n}(s)\right)^{k} \text { for } \mathrm{n} \neq 0
$$

and

$$
\psi_{* 0}^{+(k)}(s)=\left(\psi_{* 0}(s)\right)^{k}
$$

where we always take $k$ odd so that $\psi_{* n}^{+(k)}(s)$ is a symmetric function of $s$ and $\psi_{* n}^{-(k)}(s)$ is an antisymmetric function of $s$. Note that, $\psi_{* n}^{ \pm(k)}(m)=\delta_{n, m}$ for $n, m \in \mathcal{Z}^{+} \cup\{0\}$.

Since each of the above functions is analytic on all $\mathcal{C}$, is integrable in absolute value along every contour parallel to the imaginary axis, and uniformly converges to zero in the imaginary direction for $\Re(s) \in[a, b]$ for any closed interval, $[a, b] \subset \mathcal{R}$, these functions satisfy the conditions of the transform inversion theorem. There is therefore some $\psi_{n}^{ \pm(k)}(a)$ such that $\psi_{* n}^{ \pm(k)}(s)$ is the Laplace transform of $\psi_{n}^{ \pm(k)}(a)$.

In fact, $\psi_{n}^{(k)} \in \mathcal{L}_{ \pm}^{2}(\mathcal{R})$ since

$$
\left\|\psi_{n}^{ \pm(k)}\right\|_{\mathcal{L}^{2}}^{2}=\int_{-\infty}^{\infty} d a\left|\psi_{n}^{ \pm(k)}(a)\right|^{2}=\int_{-\infty}^{\infty} d x\left|\tilde{\psi}_{n}^{ \pm(k)}(x)\right|^{2}=\int_{-\infty}^{\infty} d x\left|\psi_{* n}^{ \pm(k)}(-i x)\right|^{2}
$$

We can estimate these norms by using the fact that for $n \geq 1$ and all real $x$,

$$
\left|\tilde{\psi}_{n}^{ \pm(k)}(x)\right| \leq e^{-k \alpha n^{2}}[Q(x)]^{k}
$$

where for convenience we have set

$$
Q(x)=2 e^{-\alpha x^{2}} e^{2 \pi|x|} / \pi^{2} \leq \frac{2 e^{\pi^{2} / \alpha}}{\pi^{2}}
$$

Let's choose $\alpha>\pi^{2}$ so that $\left|\tilde{\psi}_{n}^{ \pm(k)}\right| \leq e^{-\alpha n^{2}}<1$. Note that

$$
\left\|Q^{k}\right\|_{\mathcal{L}^{2}}<\frac{8^{k}}{\pi^{4 k}} \frac{e^{2 \pi^{2} / k \alpha} \sqrt{\pi}}{\sqrt{2 \alpha k}}<\frac{1}{\sqrt{k}}
$$

and $\psi_{n}^{ \pm(k)} \rightarrow 0$ as $k \rightarrow \infty$ as promised. For $n=0$, we make use of the facts that

$$
\frac{\sinh y}{y} \leq \frac{e^{|y|}}{1+|y|}
$$

and

$$
\frac{e^{\pi|y|}}{1+\pi|y|} \leq e^{\pi^{2}|y|^{2}}
$$


for all real $y$. These show that, for all real $x$,

$$
\left|\tilde{\psi}_{0}^{+(k)}(x)\right| \leq e^{-k\left(\alpha-\pi^{2}\right) x^{2}}
$$

and therefore that $\left\|\psi_{0}^{+(k)}\right\|_{\mathcal{L}^{2}}^{2}<\sqrt{\frac{\pi}{2 k\left(\alpha-\pi^{2}\right)}}$ and $\psi_{0}^{+(k)} \rightarrow 0$ as $k \rightarrow \infty$. For further convenience, let's choose $\alpha>\pi^{2}+1$ so that we may write simply

$$
\left\|\psi_{n}^{ \pm(k)}\right\|_{\mathcal{L}^{2}}^{2}<\frac{e^{-2 \alpha n^{2} k}}{\sqrt{k}}
$$

for all $n \in \mathcal{Z}^{+} \cup\{0\}$.

Note that this is already enough to show that $\psi_{n}^{ \pm(1)} \in \overline{K_{ \pm}\left(\mathbf{L}^{1}\right)}$, since $\psi_{n}^{ \pm(1)}-\psi_{n}^{ \pm(k)} \rightarrow \psi_{n}^{ \pm(1)}$ as $k \rightarrow \infty$ and $\psi_{n}^{ \pm(1)}-\psi_{n}^{ \pm(k)} \in K_{ \pm}\left(\mathbf{L}^{1}\right)$. This is somewhat short of our goal, but illustrates the intermediate result that the kernel is not closed. We also see that $\mathbf{L}^{1}$ is not continuous (even on $\mathcal{D}_{ \pm \mathbf{L}^{1}}$ ) as a map from $\mathcal{D}_{ \pm \mathbf{L}^{1}}$ with the $\mathcal{L}^{2}$ topology to functions of integers with the topology of pointwise convergence. This accounts for many of the strange features of the loop transform.

We are now prepared to construct the function $\psi_{*}^{\Lambda^{ \pm},(k)}$ that will be shown to take on the same values as $\Lambda_{*}^{ \pm}(s)$ at integer points for a continuous function $\Lambda^{ \pm}(a)$ of compact support $\left[3\right.$. For any such $\Lambda^{ \pm} \in \mathcal{L}_{ \pm}^{2}(\mathcal{R})$, consider the function

$$
\psi_{*}^{ \pm \Lambda,(k)}(s) \equiv \sum_{m=0}^{\infty} \hat{\Lambda}^{ \pm}(m) \psi_{* m}^{ \pm(k)}(s)
$$

defined for all $s \in \mathcal{C}$ for which this sum converges pointwise. Our first task will be to show that this sum actually converges pointwise for all complex $s$. Now, $\Lambda^{ \pm}$has compact support that must lie in some $(-L, L) \subset \mathcal{R}$. By continuity and compactness of the support, $\left|\Lambda^{ \pm}\right|$takes on some maximal value, $\Lambda_{\max }^{ \pm}$. Thus,

$$
\left|\hat{\Lambda}^{ \pm}(m)\right| \leq 2 L \Lambda_{\max }^{ \pm} e^{|m| L}
$$

If we use

$$
\left|\frac{\sin (\pi z)}{\pi z}\right| \leq \frac{\sinh (\pi|z|)}{\pi|z|} \leq e^{\pi|z|}
$$

for all complex, $z=s+i t$, we find an estimate of the magnitude of $\psi_{* n}^{ \pm(k)}(s+i t)$ :

$$
\left|\psi_{* n}^{ \pm(k)}(s+i t)\right| \leq 2^{k} e^{-k \alpha n^{2}} e^{k(\alpha s+2 \pi|s|)} e^{k\left(-\alpha t^{2}+2 \pi|t|\right)} e^{2 \pi k n}
$$

for all $s, t \in \mathcal{R}$. It follows that the sum in A12 converges for all complex $s$ and does so uniformly in any compact region, $K \subset \mathcal{C}$. In fact,

$$
\left|\psi_{*}^{\Lambda_{ \pm},(k)}(s)\right| \leq 2^{k+1} L \Lambda_{\max }^{ \pm}\left[\exp \left(\frac{(2 \pi k+L)^{2}}{2 \alpha k}\right)+1\right] e^{k\left(\alpha s^{2}+2 \pi|s|\right)} e^{k\left(-\alpha t^{2}+2 \pi|t|\right)} \sqrt{\pi}
$$

so that $\left|\psi_{*}^{\Lambda^{ \pm},(k)}(s)\right|$ is integrable along every contour parallel to the imaginary axis and satisfies the limit condition of the transform inversion theorem.

In order to use the transform inversion theorem, all that remains is to show that $\psi_{*}^{\Lambda^{ \pm},(k)}(s)$ is analytic on the entire complex plane. We already know that this function is defined for all complex $s$, so we need only show that it satisfies the Cauchy-Riemann equations. Since the sum in A12 converges uniformly in every compact subset of the complex plane, we can take derivatives of $\psi_{*}^{\Lambda^{ \pm},(k)}(s)$ term by term in the sum so long as the sum of the derivatives converges for all complex $s$. Use of our three useful facts shows that, for $n>2 s$.

\footnotetext{
${ }^{13}$ We will choose $\Lambda^{ \pm} \in \mathcal{C}_{0 \pm}^{0}(\mathcal{R})$
} 


$$
\left|\frac{d}{d s} \psi_{* m}^{ \pm(1)}\right|<2 \alpha|s| \psi_{* m}^{ \pm(1)}+4 e^{-\alpha n^{2}} e^{\alpha s^{2}} e^{2 \pi|s|} e^{2 \pi n}\left(\frac{4 e^{|s|}}{\pi}\right)
$$

so that (using Eq. A14 as well) the sum converges absolutely for all complex $s$. Since each $\psi_{* m}^{ \pm(k)}(s)$ satisfies the Cauchy-Riemann equations for all complex $s$, so does $\psi_{*}^{\Lambda^{ \pm}(k)}$, and $\psi_{*}^{\Lambda^{ \pm}(k)}$ is a holomorphic function on $\mathcal{C}$. We see that $\psi_{*}^{\Lambda^{ \pm}(k)}$ satisfies all of the conditions of the transform inversion theorem, so there must exist some $\psi^{\Lambda^{ \pm}(k)}$ such that the two-sided Laplace transform of $\psi^{\Lambda^{ \pm}(k)}$ is $\psi_{*}^{\Lambda^{ \pm}(k)}$. Using $\left\|\tilde{\psi}_{m}^{ \pm(k)}\right\|_{\mathcal{L}^{2}}$ to estimate $\left\|\tilde{\psi}^{\Lambda^{ \pm}(k)}\right\|_{\mathcal{L}^{2}}$ and thus $\left\|\psi^{\Lambda^{ \pm}(k)}\right\|_{\mathcal{L}^{2}}$, we see that $\psi^{\Lambda^{ \pm}(k)} \in \mathcal{L}_{ \pm}^{2}(\mathcal{R})$ (for odd k) and that

$$
\left\|\psi^{\Lambda^{ \pm}(k)}\right\|_{\mathcal{L}^{2}}^{2} \leq \frac{8 L^{2} \Lambda_{\max }^{ \pm 2}}{\sqrt{k}}\left(\sqrt{\frac{\pi}{4 \alpha k}} e^{L^{2} / 2 \alpha k}+1\right)
$$

which approaches zero as $k \rightarrow \infty$.

Finally, we define $\phi^{\Lambda^{ \pm}(k)} \equiv \Lambda^{ \pm}-\psi^{\Lambda^{ \pm}(k)}$, and find that $\phi^{\Lambda^{ \pm}(k)} \rightarrow \Lambda^{ \pm}$in $\mathcal{L}_{ \pm}^{2}(\mathcal{R})$ as $k \rightarrow \infty$ and

$$
\begin{aligned}
\hat{\phi}^{\Lambda^{ \pm}(k)}(n) & =\hat{\Lambda}^{ \pm}(n)-\hat{\psi}^{\Lambda^{ \pm}(k)}(n)=\hat{\Lambda}^{ \pm}(n)-\psi_{*}^{\Lambda^{ \pm}(k)}(n) \\
& =\hat{\Lambda}^{ \pm}(n)-\sum_{m=0}^{\infty} \hat{\Lambda}^{ \pm(m)} \psi_{* m}^{ \pm(k)}(n) \\
& =\hat{\Lambda}^{ \pm}(n)-\sum_{m=0}^{\infty} \hat{\Lambda}^{ \pm(m)} \delta_{m, n}=\hat{\Lambda}^{ \pm}(n)-\hat{\Lambda}^{ \pm}(n)=0
\end{aligned}
$$

so that $\phi^{\Lambda^{ \pm},(k)} \in K_{ \pm}\left(\mathbf{L}^{1}\right)$ and we see that $\mathcal{L}_{ \pm}^{2}(\mathcal{R}) \subset \overline{\mathcal{C}_{0 \pm}^{0}(\mathcal{R})} \subset \overline{K_{ \pm}\left(\mathcal{L}^{1}\right)}$ and $K_{ \pm}\left(\mathbf{L}^{1}\right)$ is dense in $\mathcal{L}_{ \pm}^{2}(\mathcal{R})$. It follows that, since $K(\mathbf{L})=K_{+}\left(\mathbf{L}^{1}\right) \otimes K_{+}\left(\mathbf{L}^{1}\right) \oplus K_{-}\left(\mathbf{L}^{1}\right) \otimes K_{-}\left(\mathbf{L}^{1}\right)$, we have

$$
\begin{aligned}
\overline{K(\mathbf{L})} & =\overline{K_{+}\left(\mathbf{L}^{1}\right)} \otimes \overline{K_{+}\left(\mathbf{L}^{1}\right)} \oplus \overline{K_{-}\left(\mathbf{L}^{1}\right)} \otimes \overline{K_{-}\left(\mathbf{L}^{1}\right)} \\
& \left.=\mathcal{L}_{(}^{2} \mathcal{R}\right) \otimes \mathcal{L}_{+}^{2}(\mathcal{R}) \oplus \mathcal{L}_{-}^{2}(\mathcal{R}) \otimes \mathcal{L}_{-}^{2}(\mathcal{R}) \\
& =\mathcal{L}_{+}^{2}\left(\mathcal{R}^{2}\right)
\end{aligned}
$$

and we have shown that the kernel of the loop transform is dense in the connection representation.

Note that the only fact about $\Lambda^{ \pm}$that we actually used in our construction of the function $\psi^{\Lambda^{ \pm},(k)}(a)$ was that $\left|\hat{\Lambda}^{p m}(m)\right| \leq 2 L \Lambda_{m a x}^{ \pm} e^{|m| L}$. It follows that given any set of numbers $\left\{\hat{\psi}_{1}(n)\right\}$ that satisfy $\hat{\psi}_{1}(n)= \pm \hat{\psi}_{1}(-n)$ and also have a bound of the form $\left|\hat{\psi}_{1}(n)\right| \leq \psi_{\max } e^{|n| L}$ for some $\psi_{\max }$ and $L$, there is some $\psi_{1} \in \mathcal{L}_{ \pm}^{2}$ such that $\left(\mathbf{L}^{1} \psi_{1}\right)(n)=$ $\hat{\psi}_{1}(n)$. If instead of working with functions of one dimensional arguments we work with functions of two dimensional arguments:

$$
\hat{\psi}(n, m)=\hat{\psi}(-n,-m) \text { and }|\hat{\psi}(n, m)| \leq \psi_{\max } e^{|n| L_{1}+|m| L_{2}}
$$

for some $\psi_{\max }, L_{1}, L_{2} \in \mathcal{R}$, we could define $\psi_{*}$ by

$$
\psi_{*}(s) \equiv \sum_{n, m=0}^{\infty} \hat{\psi}(n, m) \psi_{* n}\left(s^{1}\right) \psi_{* m}\left(s^{2}\right)
$$

by analogy with Eq. A12 then a similar calculation would show that there must be some function $\psi(\mathbf{a})$ in $\mathcal{L}_{+}^{2}(\mathcal{R})$ such that $\hat{\phi}(n, m)$ is the loop transform of $\psi(\mathbf{a})$. In particular, this shows that the image of the loop transform contains all $l^{2}$ functions of loops; that is, all of those functions that make up the standard loop representation.

\section{APPENDIX B: SOME CANDIDATE SPACES}

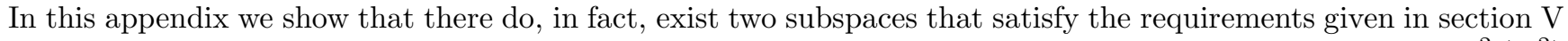
and give proofs of the properties of these subspaces that were quoted. That is, we seek linear subspaces $\mathcal{V}$ of $\mathcal{L}_{+}^{2}\left(\mathcal{R}^{2}\right)$ that satisfy:

i) $\mathcal{V}$ is dense in $\mathcal{L}_{+}^{2}$

ii) $\mathcal{V}$ is stable under $\mathcal{T}^{0}, \mathcal{T}^{1 \prime} \mathrm{s}$ 
iii) $\mathcal{V} \cap K(\mathbf{L})=\{0\}$

iv) $\mathcal{V} \subset \mathcal{D}_{\mathbf{L}}$

For the first such subspace, let $\mathcal{V}_{1}$ be the vector space consisting of all functions of the form:

$$
\begin{aligned}
\psi_{\mathbf{A}, \mathbf{B}}=e^{-|\mathbf{a}|^{2}} \sum_{\mathbf{m} \in \mathcal{Z}^{2} / \mathcal{Z}_{2}, \mathbf{n} \in \mathcal{Z}^{+} \times \mathcal{Z}^{+}} & \left(\cosh (\mathbf{m} \cdot \mathbf{a})\left(a_{1}\right)^{n_{1}}\left(a_{2}\right)^{n_{2}} A_{\mathbf{m}, \mathbf{n}}\right. \\
+ & \left.\sinh (\mathbf{m} \cdot \mathbf{a})\left(a_{1}\right)^{n_{1}}\left(a_{2}\right)^{n_{2}} B_{\mathbf{m}, \mathbf{n}}\right)
\end{aligned}
$$

where $A_{\mathbf{m}, \mathbf{n}}=0$ for $n_{1}+n_{2}$ odd and $B_{\mathbf{m}, \mathbf{n}}=0$ for $n_{1}+n_{2}$ even. The coefficients $A_{\mathbf{m}, \mathbf{n}}$ and $B_{\mathbf{m}, \mathbf{n}}$ are to be nonzero only for a finite number of pairs $\mathbf{n}, \mathbf{m}$.

To see that this space is stable under the $\mathcal{T}^{0}$ 's and $\mathcal{T}^{1}$ 's, note that taking derivatives and multiplication by cosh and sinh preserves the form described in Eq. B1 up to possible violations of the conditions on the coefficients that depend on the even or odd character of $n_{1}+n_{2}$. However, the $\mathcal{T}^{0}$ 's and $\mathcal{T}^{1}$ 's preserve $\mathcal{L}_{+}^{2}$, so these conditions must not be violated. The space of functions described by Eq. B1 is therefore invariant under the action of the $\mathcal{T}^{0}$ 's and $\mathcal{T}^{1}$ 's.

We can also see that $\mathcal{V}_{1}$ is dense in $\mathcal{L}_{+}^{2}$ since it contains the (properly symmetrized) energy eigenstates of two uncoupled harmonic oscillators (i.e, the states of the form $e^{-|\mathbf{a}|^{2}} H_{n_{1}}\left(a_{1}\right) H_{n_{2}}\left(a_{2}\right)$ ) where $H_{n}$ is the Hermite polynomial of order $n$ ). The rapid fall off of $e^{-|\mathbf{a}|^{2}}$ ensures that $\mathcal{V}_{1} \subset \mathcal{D}_{\mathbf{L}}$. Thus, we need only show that $\mathcal{V}_{1}$ satisfies (iii).

To verify this final requirement, we note that the two sided Laplace transform of $\psi_{\mathbf{A}, \mathbf{B}}$ is of the form,

$$
\begin{aligned}
\psi_{\mathbf{A}, \mathbf{B}}=e^{-|\mathbf{s}|^{2} / 4} \sum_{\mathbf{m} \in \mathcal{Z}^{2} / \mathcal{Z}_{2}, \mathbf{n} \in \mathcal{Z}^{+} \times \mathcal{Z}^{+}} & \left(\cosh \left(\frac{\mathbf{m} \cdot \mathbf{s}}{2}\right) \mathcal{P}_{n_{1}}^{(c)}\left(s_{1}\right) \mathcal{P}_{n_{2}}^{(c)}\left(s_{2}\right) A_{\mathbf{m}, \mathbf{n}}\right. \\
+ & \left.\sinh \left(\frac{\mathbf{m} \cdot \mathbf{s}}{2}\right) \mathcal{P}_{n_{1}}^{(s)}\left(s_{1}\right) \mathcal{P}_{n_{2}}^{(s)}\left(s_{2}\right) B_{\mathbf{m}, \mathbf{n}}\right)
\end{aligned}
$$

where $\mathcal{P}_{n}^{(c)}(s), \mathcal{P}_{n}^{(s)}(s)$ are polynomials of degree $n$ in $s$. Any function of this form with a finite number of nonzero coefficients has a largest $m_{1}$ such that $A_{\mathbf{m}, \mathbf{n}}$ or $B_{\mathbf{m}, \mathbf{n}}$ is nonzero for some $m_{2}, n_{1}, n_{2}$ and a largest $n_{1}$ such that (for this value of $m_{1}$ and some $\left.m_{2}, n_{2}\right) A_{\mathbf{m}, \mathbf{n}}$ or $B_{\mathbf{m}, \mathbf{n}}$ is nonzero. For fixed $s_{2}$ and large enough $s_{1}$, this term will dominate and $\psi_{\mathbf{A}, \mathbf{B}}\left(s_{1}, s_{2}\right)$ is not zero unless

$$
\sum_{n_{2}=0, m_{2}=-\infty}^{\infty} e^{m_{2} s_{2} / 2}\left(\mathcal{P}_{n_{2}} \mathcal{P}_{n}^{(c)}(s)\left(s_{2}\right) A_{\mathbf{m}, \mathbf{n}} C_{1}+\mathcal{P}_{n_{2}} \mathcal{P}_{n}^{(s)}(s)\left(s_{2}\right) B_{\mathbf{m}, \mathbf{n}} C_{2}\right)=0
$$

for some non-zero $C_{1}, C_{2} \in \mathcal{R}$.

Now, there must be at least one set of values $\left(m_{1}, n_{1}\right)$ that are selected by this procedure for values of $s_{2}$ that make up an unbounded subset $S$ of $\mathcal{R}^{+}$. Consider some $s_{2}$ in this set and those values, $m_{1}$ and $n_{1}$. We now take the largest $m_{2}$ and $n_{2}$ that contribute in $\mathrm{B} 3$ and find that, for large enough $s_{2}$ in our set $S$, this term dominates and $\psi_{\mathbf{A}, \mathbf{B}}\left(s_{1}, s_{2}\right)$ is not zero unless $C_{3} A_{\mathbf{m}, \mathbf{n}}+C_{4} B_{\mathbf{m}, \mathbf{n}}=0$ for some non-zero $C_{3}, C_{4} \in \mathcal{R}$. This cannot happen since $A_{\mathbf{m}, \mathbf{n}}$ and $B_{\mathbf{m}, \mathbf{n}}$ cannot both be non-zero for the same choice of $\mathbf{n}$ by our symmetry assumption, though by construction at least one of them is non-zero. It follows that $\mathcal{V}_{1}$ satisfies all of the stated requirements.

The second example of a subspace satisfying (i)-(iv) is quite similar. Basically, it consists of the same set of functions multiplied by judicious choices of sine and cosine functions. We consider the set of functions that can be obtained from

$$
\psi_{\alpha}=\Re\left[s^{-\alpha\left(a_{1}+\frac{i}{\alpha}\right)^{2}}\right] \Re\left[s^{-\alpha\left(a_{2}+\frac{i}{\alpha}\right)^{2}}\right]
$$

for $\alpha=m / 4 \pi, m \in \mathcal{Z}^{+}$by multiplication with polynomials that are even under $\mathbf{a} \rightarrow-\mathbf{a}$ and the functions that can in turn be obtained from these by acting with any finite number of $\mathcal{T}^{0}$ 's and $\mathcal{T}^{1}$ 's. Let $\mathcal{V}_{2}$ be the set of finite linear combinations of these functions. By inspection, we see that any such function can be written in the form

$$
\begin{aligned}
\psi(\mathbf{a})=\sum_{\substack{i, k \in \mathcal{Z}^{+} \cup\{0\} \\
m \in \mathcal{Z}^{+}, j, n \in \mathcal{Z}}} C_{i, j, k, m, n}, \Re\left[\left(a_{1}\right)^{i} e^{j a_{1}} \exp \left(-\frac{m}{4 \pi}\left(a_{1}+\frac{2 i \pi}{m}\right)^{2}\right)\right] \\
\times \Re\left[\left(a_{2}\right)^{k} e^{n a_{2}} \exp \left(-\frac{m}{4 \pi}\left(a_{2}+\frac{2 i \pi}{m}\right)^{2}\right)\right]
\end{aligned}
$$


Since taking the Laplace transform is a real operation, the (two-sided) Laplace transforms of these functions are of the form

$$
\begin{array}{r}
\psi(\mathbf{a})=\sum_{\substack{i, k \in \mathcal{Z}^{+} \cup\{0\} \\
m \in \mathcal{Z}^{+}, j, n \in \mathcal{Z}}} C_{i, j, k, m, n,}, \Re\left[\mathcal{P}_{i}\left(s_{1}\right) e^{j s_{1} / 2} \exp \left(\frac{m}{16 \pi}\left(s_{1}+\frac{4 i \pi}{m}\right)^{2}\right)\right] \\
\times \Re\left[\mathcal{P}_{k}\left(s_{2}\right) e^{n s_{2} / 2} \exp \left(\frac{m}{16 \pi}\left(s_{2}+\frac{4 i \pi}{m}\right)^{2}\right)\right]
\end{array}
$$

where again $\mathcal{P}_{n}(s)$ is some polynomial of degree $n$ in $s$ (with a real leading coefficient). The term with the smallest $m$ value in $B 6$ must decay the most slowly and eventually dominate. An imaginary change of origin does not effect the argument we used to show that none of the nontrivial elements of $\mathcal{V}_{1}$ are in $K(\mathbf{L})$, so we may use it again here on the term with least $m$ to show that no nontrivial element of $\mathcal{V}_{2}$ is in $K(\mathbf{L})$.

We still need to show that $\mathcal{V}_{2}$ is dense in $\mathcal{L}_{+}^{2}$, but we know that we can generate all functions of the form:

$$
\Re\left[\mathcal{P}_{n_{1}}\left(a_{1}+\frac{4 \pi i}{m}\right) \exp \left(-\frac{m}{4 \pi}\left(a_{1}+\frac{4 \pi i}{m}\right)^{2}\right)\right] \Re\left[\mathcal{P}_{n_{2}}\left(a_{2}+\frac{4 \pi i}{m}\right) \exp \left(-\frac{m}{4 \pi}\left(a_{2}+\frac{4 \pi i}{m}\right)^{2}\right)\right]
$$

where $\mathcal{P}_{n}$ is an even polynomial of degree n. Using the convergence of the power series expansion for $e^{x^{2}}$, the rapid decay of $e^{-x^{2}}$, and the fact that $\Re\left[\exp \left(-\frac{m}{4 \pi}\left(a+\frac{4 \pi i}{m}\right)^{2}\right)\right]$ is even in $a$, we can conclude that

$$
\begin{aligned}
& \Re\left[\exp \left(k_{1}\left(a_{1}+\frac{4 \pi i}{m}\right)^{2}\right) \exp \left(-\frac{m}{4 \pi}\left(a_{1}+\frac{4 \pi i}{m}\right)^{2}\right)\right] \\
\times & \Re\left[\exp \left(k_{2}\left(a_{2}+\frac{4 \pi i}{m}\right)^{2}\right) \exp \left(-\frac{m}{4 \pi}\left(a_{2}+\frac{4 \pi i}{m}\right)^{2}\right)\right] \in \overline{\mathcal{V}}_{2}
\end{aligned}
$$

for any $k_{1}, k_{2}<\frac{m}{4 \pi}$. In particular, let $k_{1}, k_{2}=\frac{m}{4 \pi}-1$. Then we have shown that $\Re\left[\exp \left(-\left(a_{1}+\frac{4 \pi i}{m}\right)^{2}\right)\right] \Re\left[\exp \left(-\left(a_{2}+\frac{4 \pi i}{m}\right)^{2}\right)\right] \in \overline{\mathcal{V}}_{2}$. Finally, in this form we can take the limit $m \rightarrow \infty$ so that we find that $e^{-\left(a_{1}\right)^{2}} e^{-\left(a_{2}\right)^{2}} \in \overline{\mathcal{V}}_{2}$. We could similarly obtain this function multiplied by any polynomial that is even in a. The set of such functions is a dense subspace of $\mathcal{L}_{+}^{2}$ which shows that $\mathcal{V}_{2}$ is dense in $\mathcal{L}_{+}^{2}$.

Thus we see that $\mathcal{V}_{2}$ also satisfies requirements (i)-(iv). Note that it has no elements in common with $\mathcal{V}_{1}$. However, their loop transforms do have elements in common. To show this, we consider the Laplace transform of one of the functions, $\psi_{\frac{1}{4 \pi}}$, defined in $\mathrm{B} 4$ :

$$
\psi_{* \frac{1}{4 \pi}}=C \Re\left[e^{\left(s_{1}+4 i \pi\right)^{2} / 4}\right] \Re\left[e^{\left(s_{2}+4 i \pi\right)^{2} / 4}\right]=C e^{-4 \pi} e^{\left(\left(s_{1}\right)^{2}+\left(s_{2}\right)^{2}\right) / 4} \cos \left(2 \pi s_{1}\right) \cos \left(2 \pi s_{2}\right)
$$

for some $C \in \mathcal{R}$. For integer $s$, the cosines are just equal to one. Up to a constant factor, this is the same as the two-sided Laplace transform of $e^{-\left(a_{1}\right)^{2}} e^{-\left(a_{2}\right)^{2}}$, which is in $\mathcal{V}_{1}$. The images of $\mathcal{V}_{1}$ and $\mathcal{V}_{2}$ have elements in common, as was claimed in section $\mathrm{V}$. A careful calculation shows that the functions in $\mathcal{V}_{1}$ and $\mathcal{V}_{2}$ that map to the same function of loops have different $\mathcal{L}^{2}$ norms.

It was also mentioned in section $\mathrm{Q}$ that neither of these spaces have subspaces that fulfill requirements (i)-(iv) and which form an algebraically irreducible representation of the $\mathcal{T}^{0}$ 's and $\mathcal{T}^{1}$ 's. This is because acting with a $\mathcal{T}^{1}(\mathbf{k})$ increases the degree of the polynomial part of the function according to the decompositions used in Eq. B2 and Eq. B6 but no action by any combination of $\mathcal{T}^{0}$ 's and $\mathcal{T}^{1}$ 's can lower the polynomial degree of these functions. This is the last of the properties of these subspaces that was quoted in section $\mathrm{V}$.

\section{APPENDIX C: THE INNER PRODUCT IN THE CONNECTION REPRESENTATION}

This appendix serves to verify that the $\mathcal{T}^{0}$ 's and $\mathcal{T}^{1}$ 's determine the same inner product in the connection representation as a and $\mathbf{p}$. With the assumption that the connection representation forms an $\mathcal{L}^{2}$ space, we will show that Hermiticity of the $\mathcal{T}^{0}$ 's and $\mathcal{T}^{1}$ 's requires the same inner product as Hermiticity of $\mathbf{a}$ and $\mathbf{p}$ if the representation is required to be topologically irreducible. Interestingly, topological irreducibility plays a much stronger role when the inner product is determined using loop variables than when $\mathbf{a}$ and $\mathbf{p}$ are used.

In the connection representation, $\mathcal{T}^{0}(\mathbf{k})$ is represented by a multiplication operator and $\mathcal{T}^{1}(\mathbf{k})$ is represented by a differential operator. Thus, in the timelike sector, we consider an inner product defined on some set of smooth functions on the torus. Since the configuration space is a compact manifold without boundary, we will be able to perform integrations by parts in the integral that defines the inner product. Performing these integrations by parts shows that we must have

$$
\sin (\mathbf{n} \cdot \mathbf{a}) \quad \mathbf{n} \times \frac{\partial}{\partial \mathbf{a}} d \mu=0
$$


where $d \mu$ is the measure that defines the inner product, for the $\mathcal{T}^{1}(\mathbf{n})$ 's to be Hermitian. This requires the measure to be constant, except at the points $a_{b} \in\{0, \pi\}$ where it could have delta-function singularities 14 . If it does have delta function singularities at these points, then the completion of this space is $\mathcal{L}^{2} \oplus \mathcal{C}^{4}$, where the $\mathcal{C}^{4}$ is represented by characteristic functions of the four singular points. These functions are mapped into themselves by the $\mathcal{T}^{0}$ 's and $\mathcal{T}^{1}$ 's, and so form a closed invariant subspace and the representation is not topologically irreducible. The only choice that gives a topologically irreducible representation is to include none of these singularities in $d \mu$ so that $d \mu$ must be a constant.

Note that we could also build a topologically irreducible representation by starting with the set of all smooth functions on the torus that vanish at these four exceptional points. There is then no ambiguity in the measure; it must be constant. However, the set of functions that vanish at a discrete set of point is dense in $\mathcal{L}^{2}$, so the completed space is the same as the one given by the above argument, $\mathcal{L}^{2}\left(\mathcal{T}^{2}\right)$.

The same argument can be performed in the spacelike sector by replacing $\sin (\mathbf{n} \cdot \mathbf{a})$ with $\sinh (\mathbf{n} \cdot \mathbf{a})$ in the above discussion and by considering an initial set functions on the configuration space that contains all smooth functions that fall off sufficiently rapidly near infinity. In this case, only the origin appears as an exceptional point so that there is only one free parameter in the measure corresponding to the strength of a delta-function singularity at the origin. If this parameter is not set to zero, the completion of our initial set of functions yields $\mathcal{L}^{2} \oplus \mathcal{C}$, which is again topologically reducible. The only topologically irreducible choice is to set this parameter to zero and to find that the completed Hilbert space is just $\mathcal{L}^{2}$. Again, we would uniquely arrive at this space without explicitly imposing topological irreducibility if we started with a space of smooth functions that vanish at the exceptional point.

\section{APPENDIX D: THE UNIQUENESS OF THE LOOP REPRESENTATION}

In this appendix we will show that there is a unique definition of the loop representation and a unique choice of inner product for this representation such that

i) The loop representation contains at least one function with support on a single loop.

ii) The function of loops ${ }^{15}$ that maps all loops to zero is the zero state.

iii) The $\mathcal{T}^{0}$ 's and $\mathcal{T}^{1}$ 's are Hermitian with respect to the inner product.

iv) The loop representation is a topologically irreducible representation of the $\mathcal{T}^{0}$ 's and $\mathcal{T}^{1}$ 's.

We will begin by showing that our loop representation $\mathcal{R}$ contains all functions with support on a finite number of loops. Note that we are guaranteed by (i) to have at least one such function that in fact has support on only one loop, $\mathbf{n}$. Let's denote by $|\mathbf{n}\rangle$ the function that takes the value one on this loop and zero on all others. We similarly define $|\mathbf{k}\rangle$ for all other loops, $\mathbf{k}$, although at this point we do not know if $\mathcal{R}$ contains any of these other functions.

To show that these other functions with support on single loops are in $\mathcal{R}$, we consider the action of the operator $\frac{1}{2}\left(\mathcal{T}^{1}(\mathbf{k})+i\left(\mathbf{n} \times \mathbf{k} \mathcal{T}^{0}(\mathbf{k})\right)\right.$ on $|\mathbf{n}\rangle:$

$$
\frac{1}{2}\left(\mathcal{T}^{1}(\mathbf{k})+i\left(\mathbf{n} \times \mathbf{k} \mathcal{T}^{0}(\mathbf{k})\right)|\mathbf{n}\rangle=i(\mathbf{n} \times \mathbf{k})|\mathbf{n}+\mathbf{k}\rangle\right.
$$

Since $R$ is a representation of the $\mathcal{T}^{0}$ 's and $\mathcal{T}^{1}$ 's, this implies that for $\mathbf{k} \times \mathbf{n} \neq 0 R$ contains $|\mathbf{n}+\mathbf{k}\rangle$. However, $\mathbf{k} \times \mathbf{n} \neq 0$ is equivalent to $(\mathbf{k}-\mathbf{n}) \times \mathbf{n} \neq 0$ so that for $\mathbf{k} \times \mathbf{n} \neq 0 R$ contains $|\mathbf{k}\rangle$. If we choose some such $\mathbf{k}$, then $R$ must also include the state $|2 \mathbf{k}\rangle$ and therefore the state:

$$
\mathcal{T}^{0}(\mathbf{k})|\mathbf{k}\rangle-\frac{1}{2}|2 \mathbf{k}\rangle=\frac{1}{2}|\mathbf{0}\rangle
$$

so that the characteristic function of the zero loop is a member of our representation. Since $\mathcal{T}^{0}(\mathbf{k})|\mathbf{0}\rangle=|\mathbf{k}\rangle$, all characteristic functions of loops and therefore all functions of loops with finite support are elements of our loop representation.

To show the uniqueness of the inner product in the loop representation, we will need a few simple relations that follow from the definitions of the $\mathcal{T}^{0}$ 's and $\mathcal{T}^{1}$ 's. We will denote the inner product of $|\mathbf{n}\rangle$ and $|\mathbf{m}\rangle$ by $\langle\mathbf{n} \mid \mathbf{m}\rangle$. It follows that, if the $\mathcal{T}^{0}(\mathbf{k})$ 's are hermitian:

$$
\langle\mathbf{m} \mid \mathbf{n}\rangle=\left\langle\mathbf{0}\left|\mathcal{T}^{0}(\mathbf{m})\right| \mathbf{n}\right\rangle=\frac{1}{2}[\langle\mathbf{0} \mid \mathbf{n}+\mathbf{m}\rangle+\langle\mathbf{0} \mid \mathbf{n}-\mathbf{m}\rangle]
$$

\footnotetext{
${ }^{14}$ Once again, thanks to Jorma Louko for pointing this out.

${ }^{15}$ In this appendix the word "loop" will again be used to refer to equitopy classes of loops.
} 
so that all inner products of states which are given by functions of loops are determined by the inner products of the states $|\mathbf{n}\rangle$ with the state $|\mathbf{0}\rangle$.

Also note that $\mathcal{T}^{1}(\mathbf{k})$ annihilates $|\mathbf{0}\rangle$ so that if $\mathcal{T}^{1}(\mathbf{k})$ is Hermitian

$$
0=\left\langle\mathbf{0}\left|\mathcal{T}^{1}(\mathbf{n})\right| \mathbf{m}\right\rangle=i \mathbf{n} \times \mathbf{m}(\langle\mathbf{0} \mid \mathbf{n}+\mathbf{m}\rangle-\langle\mathbf{0} \mid \mathbf{n}-\mathbf{m}\rangle)
$$

and that if $\mathbf{n}$ and $\mathbf{m}$ are not parallel as vectors in $\mathcal{Z}^{2}$ we have

$$
\langle\mathbf{0} \mid \mathbf{n}+\mathbf{m}\rangle=\langle\mathbf{0} \mid \mathbf{m}-\mathbf{n}\rangle
$$

We can use this equation to calculate all inner products of the form $\langle\mathbf{n} \mid \mathbf{0}\rangle$. To do so, note that any $\mathbf{n} \neq 0$ can be written in the form $\mathbf{n}=\mathbf{k}+(a, b)$ where $\mathbf{k} \in 2 \mathcal{Z} \times 2 \mathcal{Z}$ and $(a, b) \in\{(0,1),(1,0),(1,1),(0,2)\}$. If we consider some $\mathbf{n}$ that is not proportional to its corresponding $(a, b)$ then, from Eq. D5 we find that

$$
\langle\mathbf{0} \mid \mathbf{n}\rangle=\left\langle\mathbf{0} \mid \frac{\mathbf{n}+(a, b)}{2}+\frac{\mathbf{n}-(a, b)}{2}\right\rangle=\left\langle\mathbf{0} \mid \frac{\mathbf{n}+(a, b)}{2}-\frac{\mathbf{n}-(a, b)}{2}\right\rangle=\langle\mathbf{0} \mid(a, b)\rangle
$$

If, on the other hand, $\mathbf{n}$ is proportional to its $(a, b)$ we can perform this argument replacing $(a, b)$ with $\left(a^{\prime}, b^{\prime}\right)=$ $(a, b)+(4,2)$ to which $\mathbf{n}$ will not then be proportional. In this case, $\langle\mathbf{0} \mid \mathbf{n}\rangle=\left\langle\mathbf{0} \mid\left(a^{\prime}, b^{\prime}\right)\right\rangle$. But, by the above argument, $\langle\mathbf{0} \mid(a, b)\rangle=\left\langle\mathbf{0} \mid\left(a^{\prime}, b^{\prime}\right)\right\rangle$. If we fix the normalization of the zero loop to be $\langle\mathbf{0} \mid \mathbf{0}\rangle=1$, we find that the inner products are determined by the four parameters $\langle\mathbf{0} \mid(a, b)\rangle$.

Under the Fourier transform map from sequences to functions on the torus, these four coefficients are just combinations of the strengths of the four singularities allowed in the measure, $d \mu$, of the connection representation of the timelike sector that were found in Appendix C. In terms of loop coefficients, these parameters produce terms in the inner product $\left\langle\psi_{1} \mid \psi_{2}\right\rangle$ that depend on $\sum_{\mathbf{n} \in 2 \mathcal{Z} \times 2 \mathcal{Z}+(a, b)} \psi_{1}(\mathbf{n})$ and $\sum_{\mathbf{n} \in 2 \mathcal{Z} \times 2 \mathcal{Z}+(a, b)} \psi_{2}(\mathbf{m})$. The subspace such that this sum is zero for all $(a, b)$ is invariant under the action of the $\mathcal{T}^{0}$ 's and $\mathcal{T}^{1}$ 's (in fact, it contains the image of every $\left.\mathcal{T}^{1}(\mathbf{k})\right)$. When this term enters into the inner product with non-zero coefficient, this subspace is closed and the representation is not topologically irreducible 16 . Thus, if the representation is to be topologically irreducible, these four parameters must be zero and the inner product is unique. This is just the result that would be anticipated from Ref. [27].

Since the inner products of the states $|\mathbf{n}\rangle$ are unique, $R$ must contain the completion in this inner product of the space of functions with support on a finite number of loops. With the parameters $\langle\mathbf{0} \mid(a, b)\rangle$ set to zero, we see from Eq. D3 that $\langle\mathbf{n} \mid \mathbf{m}\rangle=\frac{1}{2} \delta_{\mathbf{n}, \mathbf{m}}$ for $\mathbf{n}$ or $\mathbf{m}$ not zero and $\langle\mathbf{0} \mid \mathbf{0}\rangle=1$. Therefore, this completion is just the set of $l^{2}$ functions of loops. However, this space is a closed subspace (in this topology) of $R$ and is left invariant by the $\mathcal{T}^{0}$ 's and $\mathcal{T}^{1}$ 's so that, if $R$ is indeed topologically irreducible, this space must be all of $R$. It follows that the choice of $R$ and the inner product imposed on it follow uniquely from requirements (i)-(iv).

[1] Gross D and Periwal V 1988 Phys. Rev. Lett 60 2105-8

[2] DeWitt B 1989 Preprint University of Texas

[3] Agishtein M and Maigdal A 1992 Mod. Phys. Lett. 7 1039-1061

[4] Dewitt B 1967 Phys. Rev. 160 1113-1148

[5] Ashtekar A 1986 Phys. Rev. Lett. 57 2244-7

[6] Ashtekar A 1987 Phys. Rev. D 36 1587-603

[7] Rovelli C and Smolin L 1990 Nucl. Phys. B133 80

[8] Smolin L 1992 Preprint SU-GP-92/12-2 Syracuse University

[9] Rovelli C 1991 Class. Quant. Grav. 81613

[10] Blenclowe M 1990 Nucl. Phys. B 341213

[11] Gambini R 1991 Phys. Lett. 255B 180

[12] Ashtekar A, Rovelli C, and Smolin L 1991 Phys. Rev. D 44 1740-55

[13] Ashtekar A, Hussain V, Rovelli C, Samuel J, and Smolin L 1989 Class. Quantum Grav. 6185

${ }^{16}$ Thanks to Alan Rendall for pointing this out. 
[14] Ashtekar A and Romano J 1989 Phys. Lett. 229B 56

[15] Louko J and Marolf D 1993 In preparation

[16] Ashtekar A 1991 Lectures on Nonperturbative Canonical Gravity (Singapore: World Scientific)

[17] Ashtekar A 1992 Class. Quantum Grav. 9 1121-1150

[18] Ashtekar A and Isham C 1992 Class. Quantum Grav. 9 1433-85

[19] Kuchař K 1986 Phys. Rev. D 34 3031-3043; Phys. Rev. D 34 3044-3057

[20] Ashtekar A, Rovelli C, and Smolin L 1992 Phys. Rev. Lett. 69 237-240

[21] Bengtsson I 1989 Phys. Lett. 229B 5

[22] Widder D 1946 The Laplace Transform (Princeton: Princeton University Press)

[23] Mess G 1990 Preprint IHES/M/90/28 Institutes des Hautes Etudes Scientifiques

[24] Di Bartolo C, Gambini R, and Griego J 1992 Preprint Montevideo

[25] Di Bartolo C, Gambini R, Griego J 1993 Preprint Universidad Simón Bolívar gr-qc/9303010

[26] Di Bartolo C, Gambini R, Griego J, Leal L 1992 Preprint Montevideo

[27] Rendall A 1993 Preprint SU-GP-93/2-2 Syracuse University

[28] Goldberg J.N., Lewandowski J, and Stornaiolo C 1992 Commun. Math. Phys. 148 377-402

[29] Loll R 1993 Preprint SU-GP-93/3-2 Syracuse University

[30] Wolpert S 1983 Ann. Math. 117207 